\title{
Caloric restriction creates a metabolic pattern of chronological aging delay that in budding yeast differs from the metabolic design established by two other geroprotectors
}

\author{
Karamat Mohammad ${ }^{1}$ and Vladimir I. Titorenko ${ }^{1}$ \\ ${ }^{1}$ Department of Biology, Concordia University, Montreal, Quebec H4B 1R6, Canada \\ Correspondence to: Vladimir I. Titorenko, email: vladimir.titorenko@concordia.ca \\ Keywords: cellular aging; geroprotectors; caloric restriction; metabolism; methionine \\ Received: January 11, $2021 \quad$ Accepted: March 15, $2021 \quad$ Published: March 30, 2021 \\ Copyright: @ 2021 Mohammad and Titorenko. This is an open access article distributed under the terms of the Creative Commons \\ Attribution License (CC BY 3.0), which permits unrestricted use, distribution, and reproduction in any medium, provided the original author \\ and source are credited.
}

\section{ABSTRACT}

Caloric restriction and the tor1 $1 \Delta$ mutation are robust geroprotectors in yeast and other eukaryotes. Lithocholic acid is a potent geroprotector in Saccharomyces cerevisiae. Here, we used liquid chromatography coupled with tandem mass spectrometry method of non-targeted metabolomics to compare the effects of these three geroprotectors on the intracellular metabolome of chronologically aging budding yeast. Yeast cells were cultured in a nutrient-rich medium. Our metabolomic analysis identified and quantitated 193 structurally and functionally diverse water-soluble metabolites implicated in the major pathways of cellular metabolism. We show that the three different geroprotectors create distinct metabolic profiles throughout the entire chronological lifespan of $S$. cerevisiae. We demonstrate that caloric restriction generates a unique metabolic pattern. Unlike the tor $1 \Delta$ mutation or lithocholic acid, it slows down the metabolic pathway for sulfur amino acid biosynthesis from aspartate, sulfate and 5-methyltetrahydrofolate. Consequently, caloric restriction significantly lowers the intracellular concentrations of methionine, $\mathbf{S}$-adenosylmethionine and cysteine. We also noticed that the low-calorie diet, but not the tor1s mutation or lithocholic acid, decreases intracellular ATP, increases the ADP:ATP and AMP:ATP ratios, and rises intracellular ADP during chronological aging. We propose a model of how the specific remodeling of cellular metabolism by caloric restriction contributes to yeast chronological aging delay.

\section{INTRODUCTION}

A body of evidence indicates that metabolism is an essential contributor to the aging and longevity of eukaryotic organisms across phyla. Indeed, healthy aging of the evolutionarily distant eukaryotes coincides with age-related changes in the concentrations of specific metabolites within cells, tissues, organs and biological fluids [1-15]. These changes are considered metabolic biomarkers characteristic of an aging-associated deterioration in cellular functionality, tissue and organs homeostasis, and organismal health [1-15]. Furthermore, such dietary interventions as caloric restriction (CR), reduced protein intake, a limited supply of single amino acid and, alternating cycles of feeding and fasting are robust geroprotectors that specifically rewire cellular and organismal metabolism in various eukaryotic organisms [16-34]. Moreover, allelic variants of the genes implicated in diverse metabolic pathways delay aging and extend longevity in eukaryotic organisms across species [3, 5, 6, 8, 9, 11, 14, 35-43]. Besides, pharmacological interventions that target distinct aspects of metabolism are potent geroprotectors in diverse eukaryotes; these interventions include metformin, rapamycin, resveratrol, spermidine and others $[36,44-$ 58]. Additionally, it has been emphasized that each of the nine common denominators (hallmarks) of aging is linked to a specific remodeling of metabolism $[59,60]$. These aging hallmarks include the damage and repair of nuclear DNA, shortening of telomeres, epigenetic regulation changes, proteotoxic stress, deregulation of nutrient sensing, deterioration of mitochondrial 
functionality, cellular senescence, decline in stem cell number and functionality, and changes in intercellular communications [59]. Based on all these observations, the existence of a metabolic "clock," "signature," "footprint" or "profile" of aging delay has been proposed [2, 4-6, 8, 20, 59].

It remained unclear if different dietary, genetic and pharmacological anti-aging interventions set up a similar metabolic pattern of aging delay or each of them generates a distinct metabolic profile. In this study, we used nontargeted metabolomics of chronologically aging budding yeast to clarify this issue. We show that three different geroprotectors create distinct metabolic profiles. We identified a unique metabolic pattern established by one of these geroprotectors.

\section{RESULTS}

\section{CR, the tor1 14 mutation and LCA extend the longevity of chronologically aging yeast}

We investigated how efficiently $\mathrm{CR}$, the tor1 $1 \Delta$ mutation and LCA prolong the longevity of chronologically aging wild-type (WT) strain BY4742. WT cells were cultured in the nutrient-rich YP $(1 \%$ yeast extract and $2 \%$ peptone) medium supplemented with glucose as a sole carbon source. Our previous studies showed that a yeast culture in this nutrient-rich medium provides a beneficial model system for elucidating the chronological aging of multicellular eukaryotes [61].

WT strain culture in the YP medium that initially contained $2 \%(\mathrm{w} / \mathrm{v})$ glucose served as a control nonCR culture for examining the CR-dependent longevity extension in chronologically aging yeast [61]. A WT strain culture in the same YP medium, but initially containing $0.2 \%(\mathrm{w} / \mathrm{v})$ glucose, was used as a model system for studying longevity extension by a CR diet [61].

The pro-longevity effect of the tor $1 \Delta$ mutation in the BY4742 genetic background was assessed in the mutant yeast cells cultured in the YP medium supplemented with $2 \%(\mathrm{w} / \mathrm{v})$ glucose. Under these conditions of culturing, the tor $1 \Delta$ mutation exhibited the highest longevity-extending efficiency [62].

LCA's greatest beneficial effect on the longevity of chronologically aging WT strain was observed if LCA was used at a final concentration of $50 \mu \mathrm{M}$ and yeast cells were cultured under CR on $0.2 \%(\mathrm{w} / \mathrm{v})$ glucose [62]. Under CR conditions on $0.2 \%(\mathrm{w} / \mathrm{v})$ glucose, a WT strain culture without LCA served as a control for elucidating the LCAdependent longevity extension.

We found that CR (a dietary geroprotective intervention), the tor $1 \Delta$ mutation (a genetic geroprotective intervention) and LCA (a pharmacological geroprotective intervention) significantly increase the mean and maximum chronological lifespans (CLS) of WT yeast cultured under the above conditions (Figure 1).

\section{CR, the tor1A mutation and LCA create different metabolic patterns throughout the entire chronological lifespan}

We used a recently developed liquid chromatography coupled with tandem mass spectrometry (LC-MS/MS) method of non-targeted metabolomics [63] to identify and quantitate the intracellular water-soluble metabolites extracted from chronologically aging yeast. Cell aliquots for the metabolomic analysis by LC-MS/ MS were collected on days 1, 2, 3, 5, 7, 10, 14, 17 and 21 of culturing (Figure 1A). A total of 193 metabolites were identified and quantitated in each of the four cultures assessed. These metabolites included AMP, ADP, ATP, $\mathrm{FAD}^{+}, \mathrm{FMN} \mathrm{FADH}_{2}, \mathrm{NAD}^{+}, \mathrm{NADH}, \mathrm{NADP}^{+}, \mathrm{NADPH}$, other nucleotides, amino acids, monosaccharides, intermediates of glycolysis and tricarboxylic cycle intermediates [63].

We found that CR causes extensive remodeling of the water-soluble metabolome within WT cells (Figure 2). The CR-dependent remodeling of the watersoluble metabolome was observed throughout the entire chronological lifespan (Figure 2). From 14\% to $43 \%$ of the identified metabolites were downregulated in WT cells recovered on different days of culturing under CR conditions (Figure 2). Culturing under CR conditions caused the upregulation of many (from $9 \%$ to $46 \%$ ) of metabolites at various stages of the aging process (Figure 2).

Akin to CR, the tor $1 \triangle$ mutation significantly altered the water-soluble metabolome of yeast (Figure 3 ). These tor1 $\Delta$-driven changes in the spectrum of intracellular water-soluble metabolites were seen on all stages of the chronological aging process in yeast cultured under nonCR conditions (Figure 3 ). The tor $1 \Delta$ mutation elicited a downregulation of $5 \%$ to $25 \%$ of all intracellular watersoluble metabolites throughout the entire chronological lifespan (Figure 3). From 17\% to 33\% of the water-soluble metabolite pool was upregulated by the tor $1 \Delta$ mutation on different days of chronological aging under non-CR conditions (Figure 3).

The intracellular water-soluble metabolome was also considerably changed by LCA (Figure 4). We detected the LCA-driven changes in the water-soluble metabolome on all days following LCA addition to WT cells under CR conditions (Figure 4). An exposure to LCA caused downregulation of $5 \%$ to $71 \%$ of all intracellular metabolites on various days after LCA addition to calorically restricted WT cells (Figure 4). Some metabolites (from $3 \%$ to $29 \%$ ) were upregulated on different days following LCA addition to these cells (Figure 4).

We normalized the data for the relative concentrations of all 193 water-soluble metabolites identified in age-matched cells cultured under CR conditions, carrying the tor $1 \Delta$ mutation or treated 
with LCA. The normalization was performed by log-transforming the data into $\log _{2}$ values of mass spectrometric peak areas for different metabolites. We used these normalized data to compare the metabolic patterns created by the three different geroprotective interventions at various stages of the chronological aging process. Multivariate analysis of the resulting data set using principal component analysis (PCA) demonstrated that the metabolic profiles of the three geroprotectors are very distinct (Figure 5). We noticed that these three geroprotector-specific metabolic profiles significantly differ from each other in yeast cells recovered on any day of culturing (Figure 5). Indeed, the metabolic patterns specific for CR, tor $1 \triangle$ and LCA were well separated from each other along the PC1 and/or PC2 axes of the PCA plots for various stages of the chronological aging process (Figure 5).

In sum, the above findings indicate that $\mathrm{CR}$, the tor1A mutation and LCA create different metabolic patterns that remain specific for a particular geroprotector throughout the entire chronological lifespan of $S$. cerevisiae.

\section{CR creates a unique pattern of the metabolic pathway for sulfur amino acid biosynthesis}

We noticed that CR, but not the torl $1 \Delta$ mutation or LCA, significantly lowers the concentration of $S$-adenosylmethionine (Sam) in yeast cells recovered on any day of culturing (Figure 6A-6C and Figure 7). Indeed,

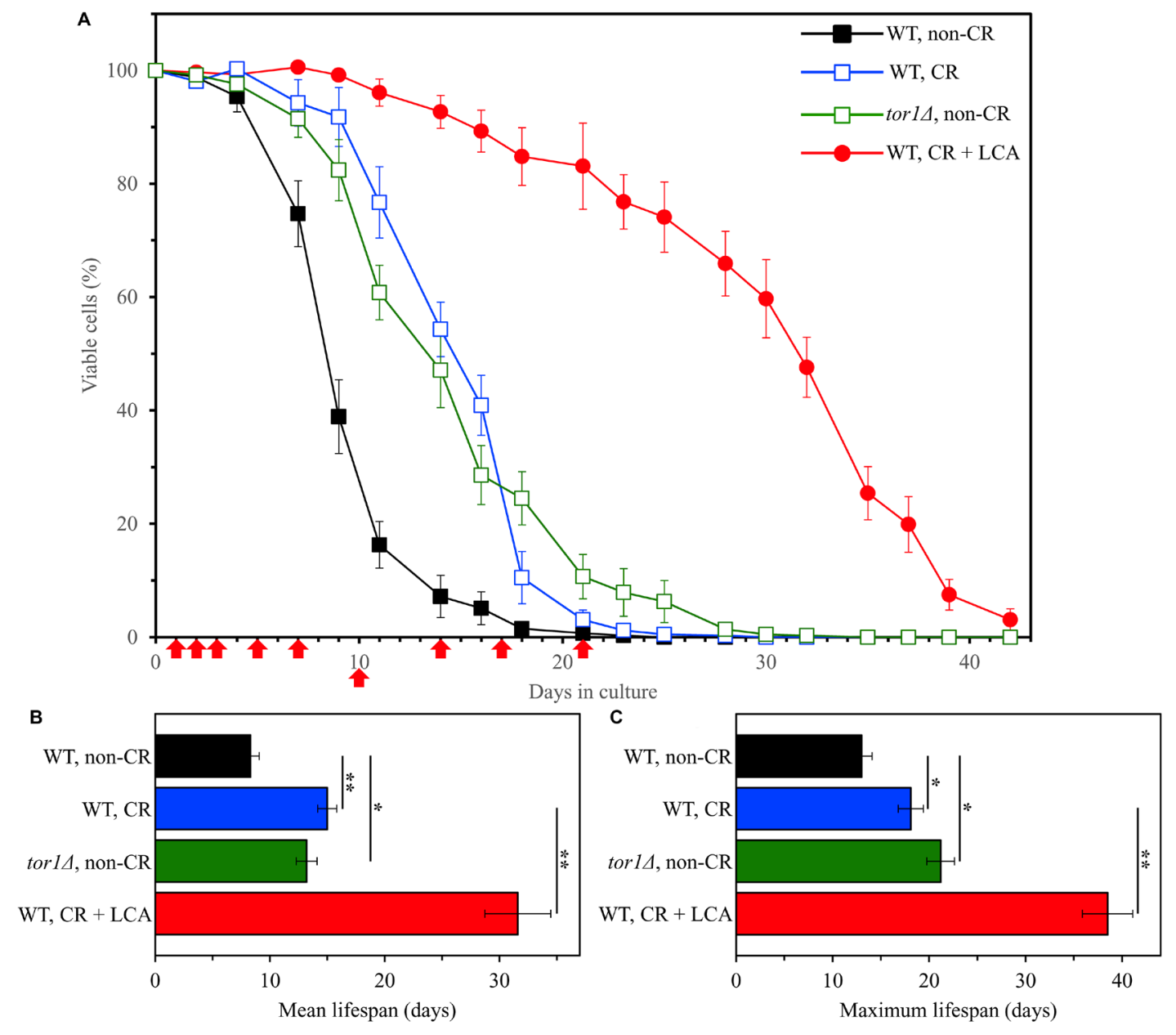

Figure 1: Caloric restriction (CR), the tor1 14 mutation and lithocholic acid (LCA) increase the chronological lifespan (CLS) of budding yeast. The wild-type (WT) strain BY4742 was cultured in the nutrient-rich YP medium initially containing 2\% (w/v) glucose (non-CR conditions), $0.2 \%(\mathrm{w} / \mathrm{v})$ glucose (CR conditions) or $0.2 \%(\mathrm{w} / \mathrm{v})$ glucose and $50 \mu \mathrm{M}$ LCA (CR + LCA conditions). The tor $1 \Delta$ mutant strain in the BY4742 genetic background was cultured in nutrient-rich YP medium initially containing $2 \%$ (w/v) glucose (nonCR conditions). Survival curves (A) and the mean and maximum lifespans (B and $\mathbf{C}$, respectively) of chronologically aging WT and torl $\Delta$ cells are shown. Data are presented as means \pm SEM $(n=3)$. In graph A, CLS extension was significant for all three longevity-extending interventions tested $(p<0.05$; the $p$ values for comparing each pair of survival curves were calculated using the logrank test described in Materials and Methods). In graphs B and C, ${ }^{*} p<0.05$ and ${ }^{* *} p<0.01$ (the $p$ values for comparing the means of two groups were calculated using an unpaired two-tailed $t$ test described in Materials and Methods). Arrows mark the time points at which cell aliquots were taken for the metabolomic analysis by LC-MS/MS. 
the intracellular concentration of Sam was decreased in yeast cultured under CR conditions throughout the entire chronological lifespan (Figure 6A and Figure 7). In contrast, Sam concentrations in yeast cells carrying the tor $1 \Delta$ mutation or treated with LCA fluctuated at various stages of the chronological aging process in a seemingly random manner (Figure 6B and 6C).

We also found that beginning of day 2 of culturing, CR causes a significant decline in methionine (Met) concentration within yeast cells at various chronological aging phases (Figure 6A and Figure 8). On the contrary, the tor $1 \Delta$ mutation and LCA elicited alterations in the intracellular concentrations of Met that randomly fluctuated on different days of cell culturing (Figure 6B and 6C).

Both Sam and Met are the two products of the metabolic pathway for sulfur amino acid biosynthesis from aspartate (Asp), sulfate and 5-methyltetrahydrofolate (5-Mtf) (Figure 6D) [64]. This pathway also leads to the biosynthesis of cysteine (Cys) (Figure 6D) [64]. We found that $\mathrm{CR}$ significantly increases the concentration of Asp throughout the entire chronological lifespan of S. cerevisiae (Figure 6A and Figure 9). CR exhibited the opposite effect on Cys concentration during most days (other than day 21) of yeast cell culturing (Figure 6A and Figure 10). Akin to the impacts of the tor $1 \Delta$ mutation and LCA on the intracellular concentrations of Sam and Met, these two geroprotectors randomly affected Asp and Cys intracellular concentrations throughout the chronological lifespan (Figure 6B and 6C).

Altogether, the above findings suggest that $\mathrm{CR}$ (but not the tor $1 \triangle$ mutation or LCA) regulates the metabolite flow along the metabolic pathway for sulfur amino acid biosynthesis in a specific manner. This geroprotective diet suppresses the biosynthesis of Met, Sam and Cys from Asp, sulfate and 5-Mtf throughout the chronological lifespan (Figure 6D).

We normalized the data on the relative concentrations of all four metabolites (i.e., Asp, Cys, Met and Sam) within the sulfur amino acid biosynthetic pathway. This normalization was performed by log-transforming the data into $\log _{2}$ values of mass
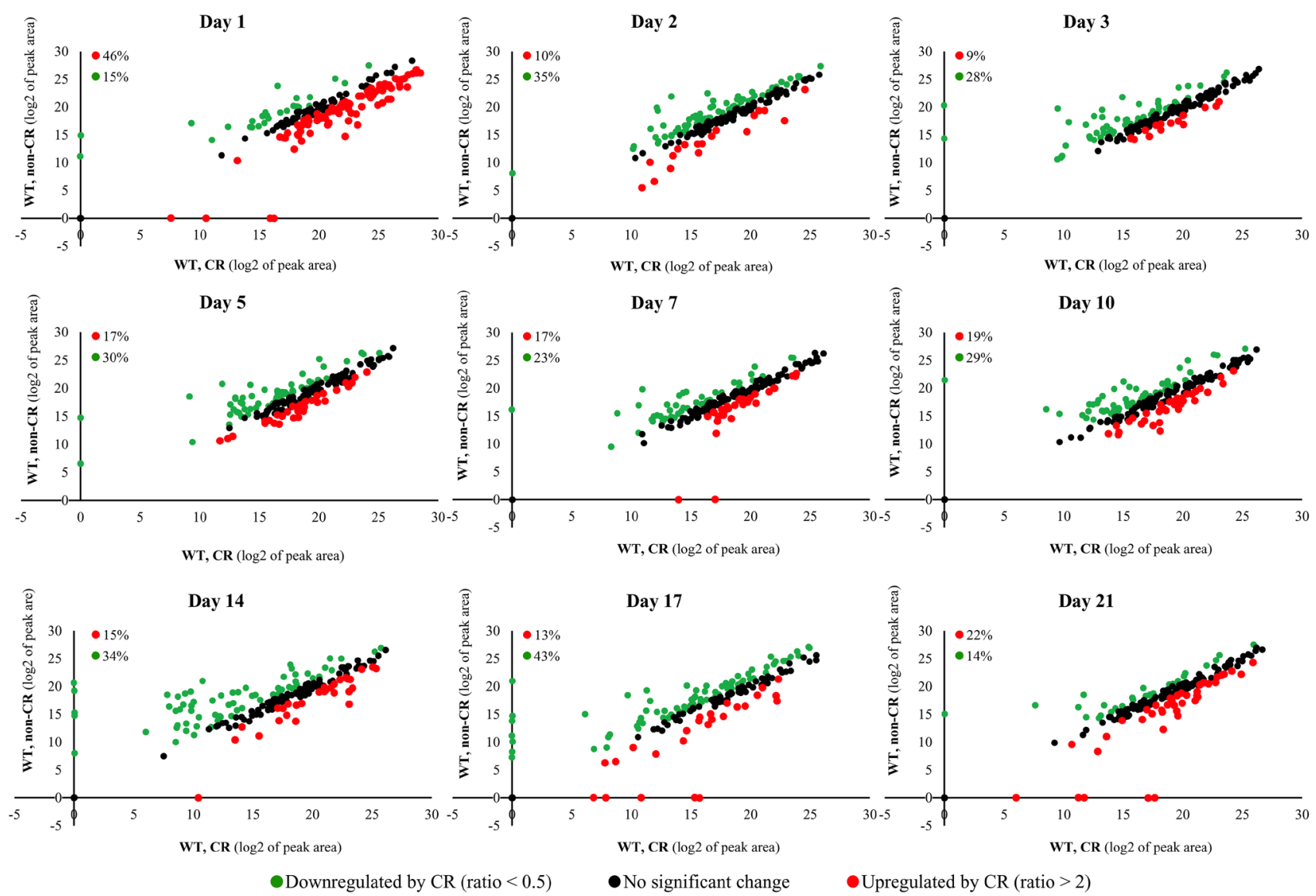

Figure 2: CR causes extensive remodeling of the water-soluble metabolome within WT cells. The WT strain BY4742 was cultured in the nutrient-rich YP medium initially containing $0.2 \%(\mathrm{w} / \mathrm{v})$ glucose (CR conditions) or 2\% (w/v) glucose (non-CR conditions). Cell aliquots for metabolic activity quenching and metabolite extraction were collected on days 1, 2, 3, 5, 7, 10, 14, 17 and 21 of culturing. The use of LC-MS/MS to identify and quantitate the intracellular water-soluble metabolites is described in Materials and Methods. Scatter plots comparing the relative abundance of water-soluble metabolites within WT cells cultured under CR or non-CR conditions are shown. The plots are on $\log _{2}-\log _{2}$ scale of mass spectrometric peak areas for different metabolites. The percentage abundance of metabolites that were upregulated (ratio $>2$; displayed in red) or downregulated (ratio $<0.5$; displayed in green) by CR is provided for each time point. 
spectrometric peak areas of the four metabolites for agematched cells cultured under CR conditions, carrying the tor1A mutation or treated with LCA. We then applied multivariate analysis by PCA to compare how the three geroprotectors affect the metabolic pathway for sulfur amino acid biosynthesis on various days of culturing. We found that the pathway patterns specific for CR, tor $1 \triangle$ and LCA are well separated from each other along the PC1 and/or PC2 axes of the PCA plots (Figure 11). Notably, these geroprotector-specific patterns were seen at diverse stages of the chronological aging process (Figure 11). We concluded that the three geroprotectors differently influence the sulfur amino acid biosynthetic pathway throughout the chronological lifespan.

\section{CR significantly increases the intracellular ADP:ATP and AMP:ATP ratios}

Three enzymatic reactions of the metabolic pathway for sulfur amino acid biosynthesis from Asp, sulfate and 5-Mtf use ATP as a co-substrate (Figure 6D) [64]. These reactions are catalyzed by Hom3p, Met7p, Sam1p and Sam $2 p$ (Figure 6D) [64]. Therefore, we expected that the CR-driven remodeling of this metabolic pathway could contribute to a change of ATP, ADP and AMP concentrations within chronologically aging yeast limited in calorie supply.

We found that CR, unlike the tor1 $1 \Delta$ mutation or LCA, significantly decreases the intracellular concentration of ATP on any day of culturing other than day 2 (Figure 6A-6C; Supplementary Figure 1). We noticed that ATP is not detected in WT cells recovered on days 17 and 21 of culturing under CR conditions without LCA or under non-CR conditions. Unlike CR, the tor $1 \triangle$ mutation and LCA caused a significant rise in ATP concentration during most cell culturing days other than day 14 and day 2, respectively (Figure 6B and 6C).

We also found that CR significantly alters (i.e., increases or decreases) the intracellular concentration of ADP during most days of yeast culturing (Figure 6A and Supplementary Figure 2). Moreover, CR increased AMP concentration within yeast cells on most chronological
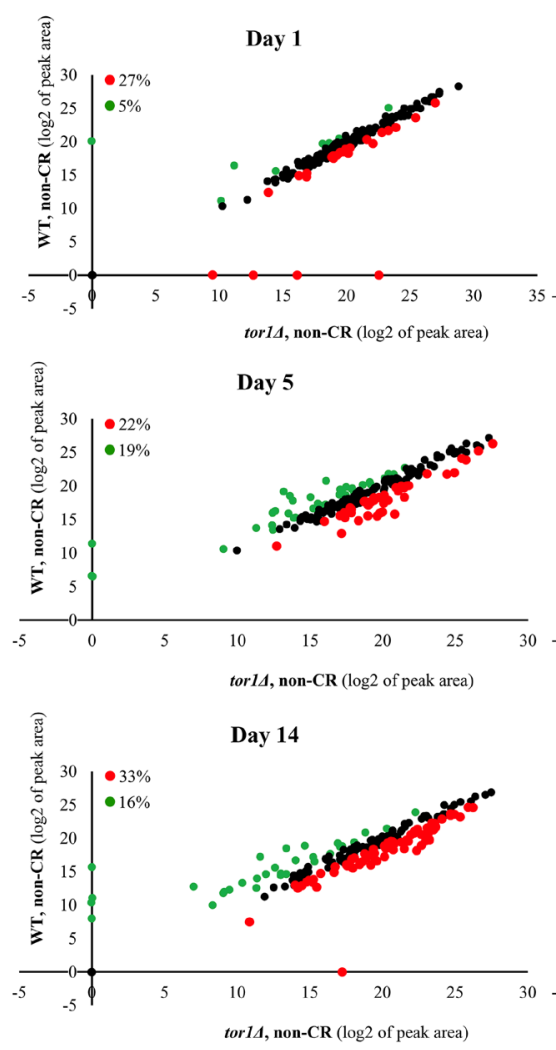

- Downregulated by tor 14 (ratio $<0.5$ )
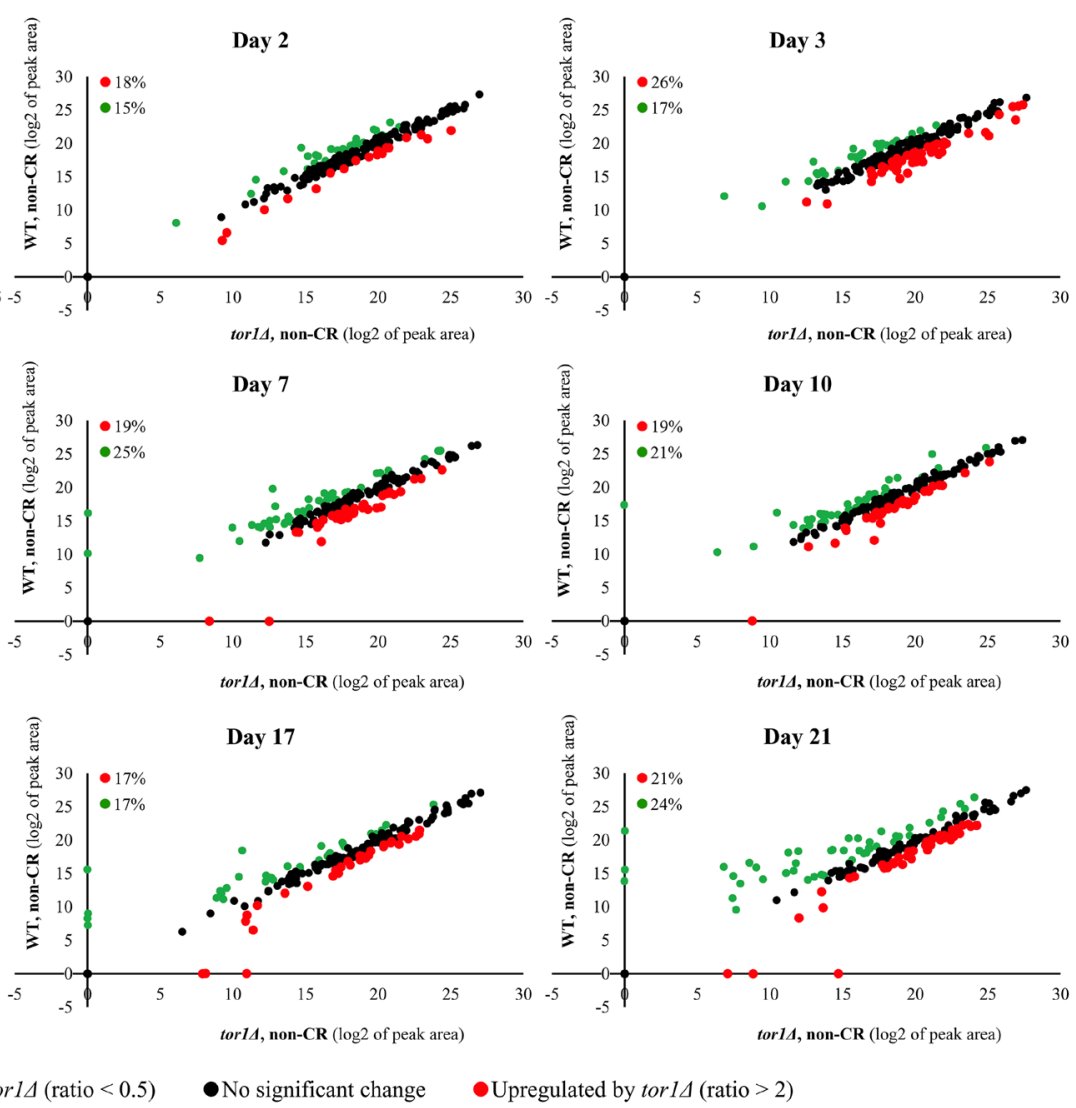

Figure 3: The tor1A mutation elicits significant changes in the water-soluble metabolome of yeast under non-CR conditions. The WT strain BY4742 and the tor $1 \Delta$ single-gene-deletion mutant strain in the BY4742 genetic background were cultured in the nutrient-rich YP medium initially containing 2\% (w/v) glucose (non-CR conditions). Cell aliquots were collected as described in the legend for Figure 2. Metabolic activity quenching, metabolite extraction, and spectrometric identification and quantitation of water-soluble metabolites were carried out as described in Materials and Methods. The data on the relative abundance of water-soluble metabolites within WT and tor $1 \Delta$ cells under non-CR conditions were plotted on a $\log _{2}-\log _{2}$ scale of mass spectrometric peak areas for different metabolites. Each plot provides the percentage abundance of metabolites that were upregulated (ratio $>2$; displayed in red) or downregulated (ratio $<$ 0.5 ; shown in green) by the tor $1 \Delta$ mutation under non-CR conditions of cell culturing. 
lifespan days (Figure 6A and Supplementary Figure 3). Unlike CR, the tor $1 \Delta$ mutation and LCA significantly decreased the intracellular concentrations of ADP and AMP at various consecutive phases of chronological aging (compare Figure 6A to Figure 6B and 6C).

We normalized the data on the concentrations of all three adenosine phosphate nucleotides (i.e., ATP, ADP and AMP) within age-matched cells cultured under CR conditions, carrying the tor 14 mutation or treated with LCA. The normalization was performed by $\log$-transforming the data into $\log _{2}$ values of mass spectrometric peak areas for the three adenosine phosphate nucleotides. We used multivariate analysis by PCA to compare the normalized data throughout the chronological lifespan. Yeast recovered on days 17 and 21 were not examined by this PCA because ATP was not detected in WT cells cultured under $\mathrm{CR}$ or non-CR conditions without LCA. Our PCA revealed that the ATP, ADP and AMP patterns specific for CR, torlA and LCA are well separated from each other along the $\mathrm{PC} 1$ and/or PC2 axes of the PCA plots (Supplementary Figure 4). Therefore, we concluded that the three geroprotectors differently affect the intracellular concentrations of adenosine phosphate nucleotides at diverse phases of yeast chronological aging.

Due to the above effects of $\mathrm{CR}$ on the relative intracellular concentrations of adenosine phosphate nucleotides, this low-calorie diet significantly increased the ADP:ATP and AMP:ATP ratios during most days of yeast cell culturing (Figure 6E, Figure 12 and Figure 13). We applied multivariate analysis by PCA to compare the ADP:ATP and AMP:ATP ratios specific for CR, torlA and LCA. Again, the data for yeast recovered on days 17 and 21 were not subject to this PCA because no ATP was detected in WT cells cultured under CR or non-CR conditions in the absence of LCA. Our PCA revealed that the normalized by log-transformation data for the ADP:ATP and AMP:ATP ratios specific for CR, tor 14 and LCA are well separated from each other along the PC1 and/or PC2 axes of the PCA plots (Supplementary Figure 5). This separation was observed for all days of cell culturing subjected to PCA (Supplementary Figure 5). Based on these findings, we concluded that the effects of
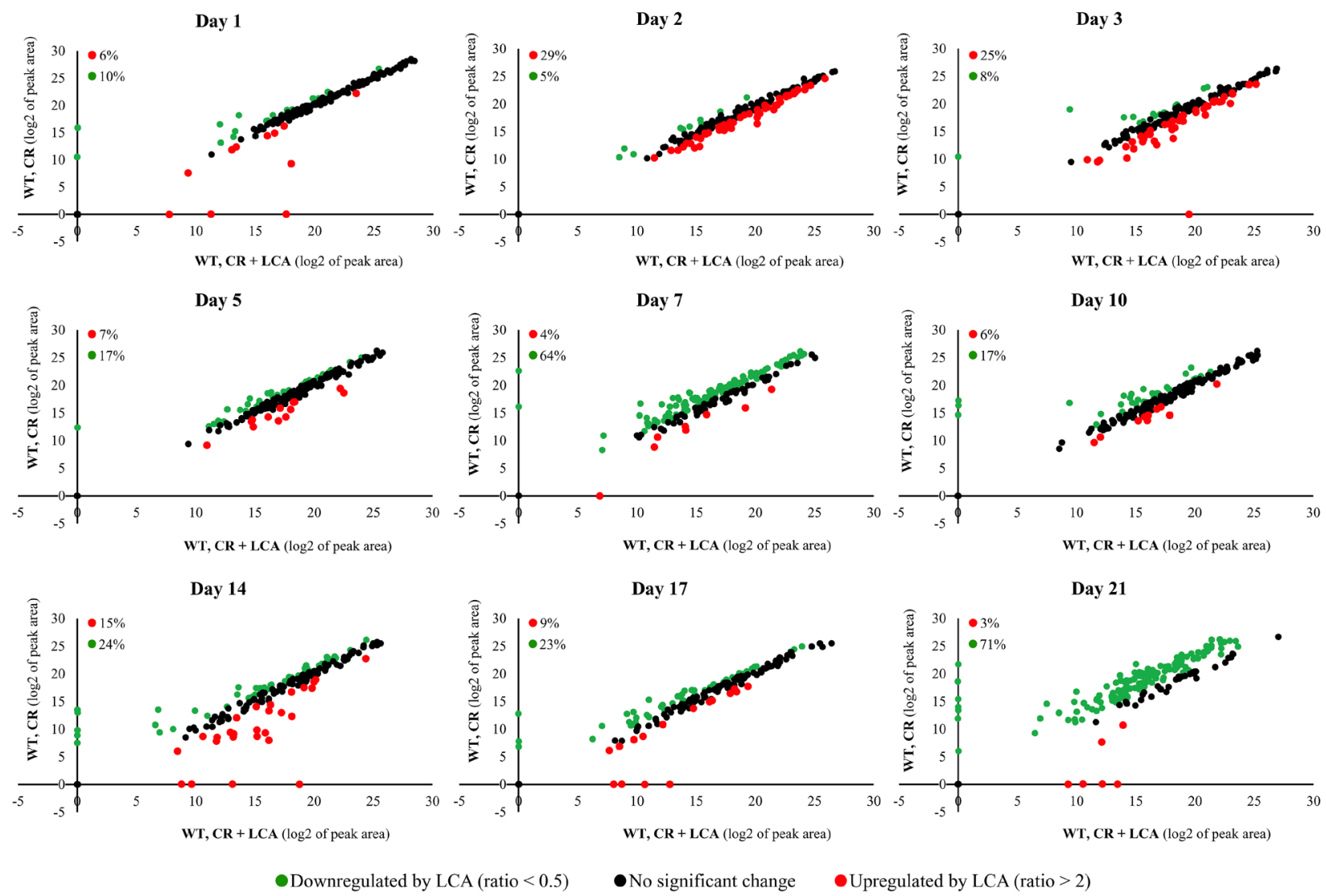

Figure 4: LCA added to calorically restricted WT cells considerably alters their intracellular water-soluble metabolome. The WT strain BY4742 was cultured in the nutrient-rich YP medium initially containing $0.2 \%$ (w/v) glucose (CR conditions), with $50 \mu \mathrm{M}$ LCA or without this bile acid. Cell aliquots were collected as described in the legend for Figure 2. The collected cells were subjected to metabolic activity quenching and metabolite extraction, followed by metabolite identification and quantitation by LC-MS/MS. All these procedures are described in Materials and Methods. Scatter plots for the relative abundance of water-soluble metabolites within WT cells cultured under CR conditions with or without LCA are presented. These plots are on a $\log _{2}-\log _{2}$ scale of mass spectrometric peak areas for different metabolites. The percentage abundance of metabolites that were upregulated (ratio $>2$; displayed in red) or downregulated (ratio $<0.5$; shown in green) by LCA is provided for each time point. 
the three geroprotectors on the intracellular ADP:ATP and AMP:ATP ratios differ throughout yeast chronological lifespan.

\section{DISCUSSION}

In this study, we used a recently developed LC-MS/ MS method of non-targeted metabolomics to investigate how three different geroprotective interventions affect the intracellular water-soluble metabolome of chronologically aging $S$. cerevisiae. These interventions include CR (a robust dietary geroprotector), the tor $1 \Delta$ mutation (an evolutionarily conserved genetic geroprotector) and LCA (a potent pharmacological geroprotector in yeast).
Our findings provide the first evidence that the three different geroprotectors create distinct metabolic patterns throughout the budding yeast's entire chronological lifespan.

It needs to be emphasized that our metabolomic analysis of the geroprotector-delayed chronological aging in yeast compared the intracellular concentrations of 193 structurally and functionally diverse watersoluble metabolites. Supplementary Table 4 provides the names of all these metabolites. AMP, ADP, ATP, FAD ${ }^{+}$, $\mathrm{FMN}, \mathrm{FADH}_{2}, \mathrm{NAD}^{+}, \mathrm{NADH}, \mathrm{NADP}^{+}, \mathrm{NADPH}$, other nucleotides, amino acids, monosaccharides, intermediates of glycolysis and tricarboxylic cycle intermediates were among these metabolites (Supplementary Table 4). Thus,
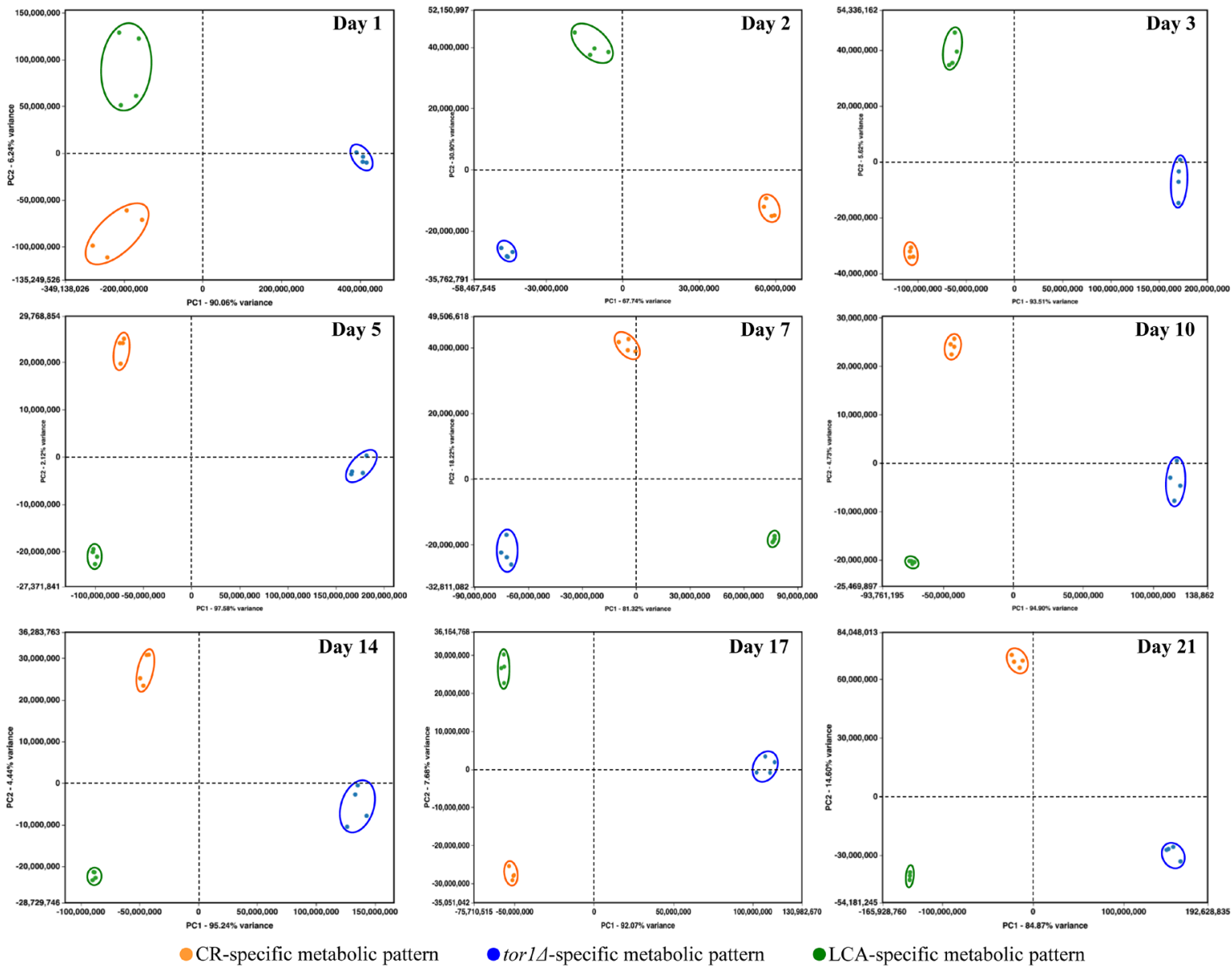

Figure 5: CR, tor14 and LCA generate different metabolic profiles of 193 water-soluble metabolites throughout the entire chronological lifespan of budding yeast. The WT strain BY4742 was cultured in the nutrient-rich YP medium initially containing $2 \%(\mathrm{w} / \mathrm{v})$ glucose (non-CR conditions), $0.2 \%(\mathrm{w} / \mathrm{v})$ glucose (CR conditions) or $0.2 \%(\mathrm{w} / \mathrm{v})$ glucose and $50 \mu \mathrm{M} \mathrm{LCA}(\mathrm{CR}+\mathrm{LCA}$ conditions). The tor $1 \Delta$ mutant strain in the BY4742 genetic background was cultured in nutrient-rich YP medium initially containing $2 \%$ $(\mathrm{w} / \mathrm{v})$ glucose (non-CR conditions). Cell aliquots for metabolic activity quenching and metabolite extraction were collected on days $1,2,3$, $5,7,10,14,17$ and 21 of culturing. The use of LC-MS/MS to identify and quantitate the intracellular water-soluble metabolites is described in Materials and Methods. A WT strain culture that initially contained 2\% (w/v) glucose served as a control non-CR culture for defining the metabolic patterns created by the CR and tor $1 \Delta$ geroprotectors. A WT strain culture that initially contained $0.2 \%(\mathrm{w} / \mathrm{v})$ glucose without LCA served as a control CR culture for defining the metabolic pattern created by the LCA geroprotector. We normalized the data for all 193 water-soluble metabolites found by log-transforming the data into $\log _{2}$ values of mass spectrometric peak areas for different metabolites. Normalized data for all these water-soluble metabolites identified in age-matched cells were used to create the principal component analysis (PCA) plots for comparing the metabolic patterns created by the three different geroprotectors. Data of 2 independent experiments, each being performed twice, are presented. 
the observed geroprotector-specific remodeling of the metabolic pattern affects the major pathways of cellular metabolism.

Our study identified a distinct metabolic pattern created by the CR geroprotector. We found two characteristic features that distinguish the CRspecific metabolic pattern from the cellular metabolism patterns created by the tor $1 \Delta$ and LCA geroprotectors. One characteristic feature that distinguishes the CRspecific metabolic design is CR's ability to suppress the biosynthesis of Met, Sam and Cys from Asp, sulfate and 5-Mtf throughout the chronological lifespan. The other characteristic feature of the CR-specific metabolic pattern is a decline in the intracellular concentration of ATP, a rise in the intracellular concentrations of ADP and AMP, and an increase in the ADP:ATP and AMP:ATP ratios at various consecutive phases of chronological aging.

Importantly, both features of the CR-specific remodeling of cellular metabolism are likely to contribute to yeast chronological aging delay by $\mathrm{CR}$.
Indeed, Met restriction is known to extend longevity in yeast and other evolutionary distant eukaryotes [65-69]. Furthermore, a recent study revealed that the met $6 \Delta$ and met $17 \Delta$ mutations (Figure 6D) decrease the intracellular concentration of Met and extend yeast replicative lifespan (RLS) under non-CR conditions in a nutrient-limited medium [70]. Moreover, the excessive quantities of exogenously added Met abrogate the CR-dependent extension of yeast RLS in a nutrient-limited medium [70]. Several mechanisms of yeast CLS extension by lowering the intracellular concentration of Met, Sam and Cys have been proposed. One likely mechanism is that a decline in intracellular Met concentration lowers the Met-dependent activation of the pro-aging Tor1 (target of rapamycin complex 1) pathway, thereby suppressing the inhibitory effect of Tor1 on the anti-aging process of autophagy [71]. Another possible mechanism is that a deterioration in the intracellular concentration of Met activates the antiaging process for proteasomal degradation of damaged and dysfunctional proteins [70]. Furthermore, a decrease
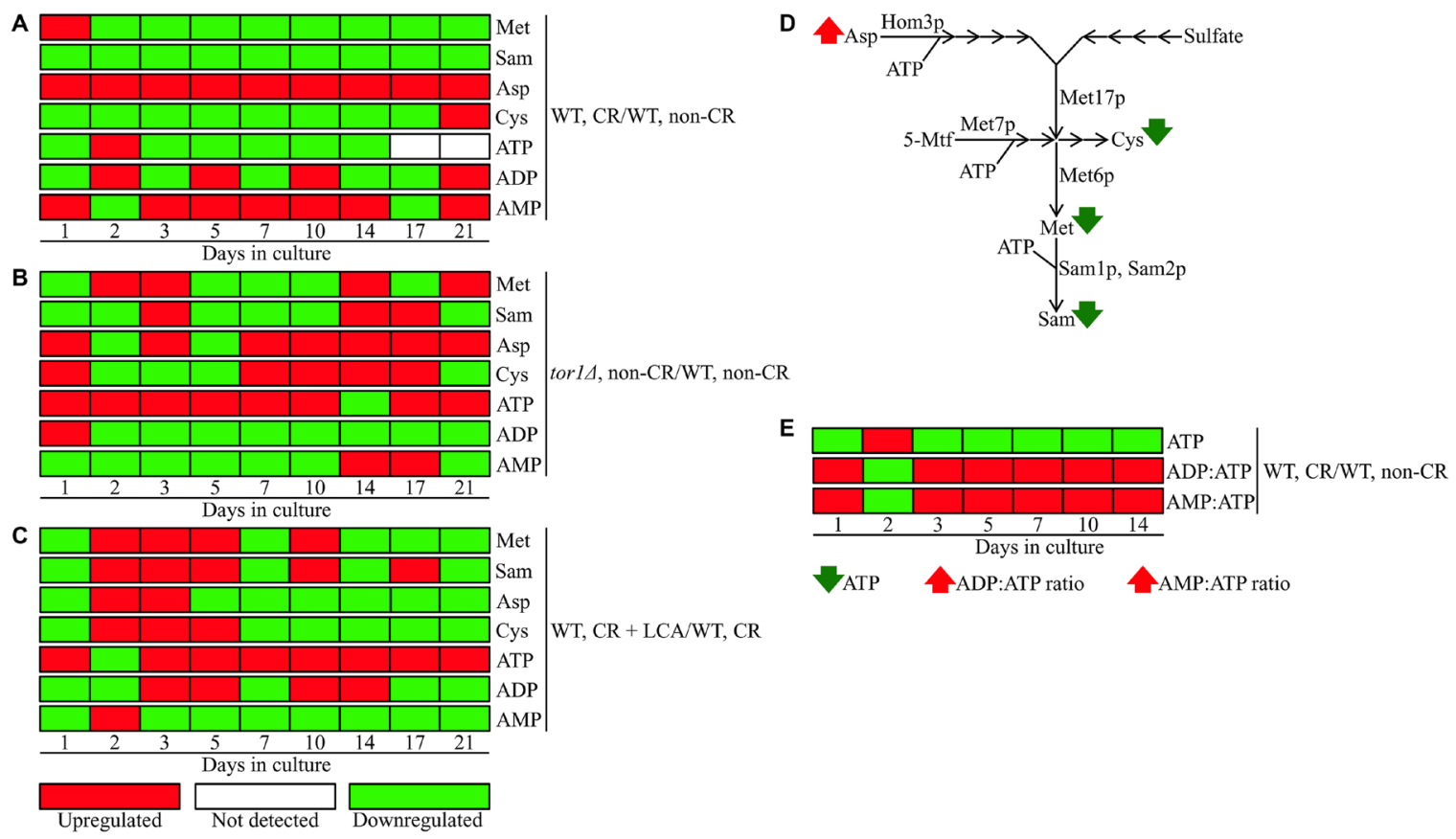

Figure 6: CR, the tor1A mutation and LCA differently influence the concentrations of methionine (Met), S-adenosylmethionine (Sam), aspartate (Asp), cysteine (Cys) and adenosine phosphate nucleotides throughout the chronological lifespan. The WT and torl 14 mutant strains were cultured, cell aliquots were collected and the metabolomic analysis by LC-MS/MS was performed as described in the legend for Figure 5. The effects of CR (A), the torl 14 mutation (B) and LCA (C) on the relative intracellular concentrations of Met, Sam, Asp, Cys, ATP, ADP and AMP are shown. CR's effect on the relative intracellular concentration of ATP and the ADP:ATP and AMP:ATP ratios are presented in E. The relative concentrations of metabolites that were significantly upregulated (displayed in red) or downregulated (shown in green) are provided for each time point. ATP was not detected in WT cells recovered on days 17 and 21 of culturing under CR or non-CR conditions without LCA. (D) A schematic depiction of how CR affects the metabolic pathway for sulfur amino acid biosynthesis from Asp, sulfate and 5-methyltetrahydrofolate (5-Mtf). (E) A graphic representation of changes in the intracellular concentration of ATP and in the ADP:ATP and AMP:ATP ratios in chronologically aging yeast limited in calorie supply. Next to metabolites' names, arrows denote those whose concentrations or ratios increase (red arrows) or decrease (green arrows) throughout most or all chronological lifespan of yeast cultured under CR conditions. Other abbreviations: Hom3p, aspartate kinase; Met6p, a cobalamin-independent methionine synthase involved (directly or indirectly) in Met and Sam biosynthesis; Met $7 \mathrm{p}$, folylpolyglutamate synthetase indirectly involved in Met, Cys and Sam biosynthesis; Met17p, an $O$-acetyl homoserine- $O$-acetyl serine sulfhydrylase indirectly involved in Met, Cys and Sam biosynthesis; Sam1p and Sam2p, S-adenosylmethionine synthetases 1 and 2. 


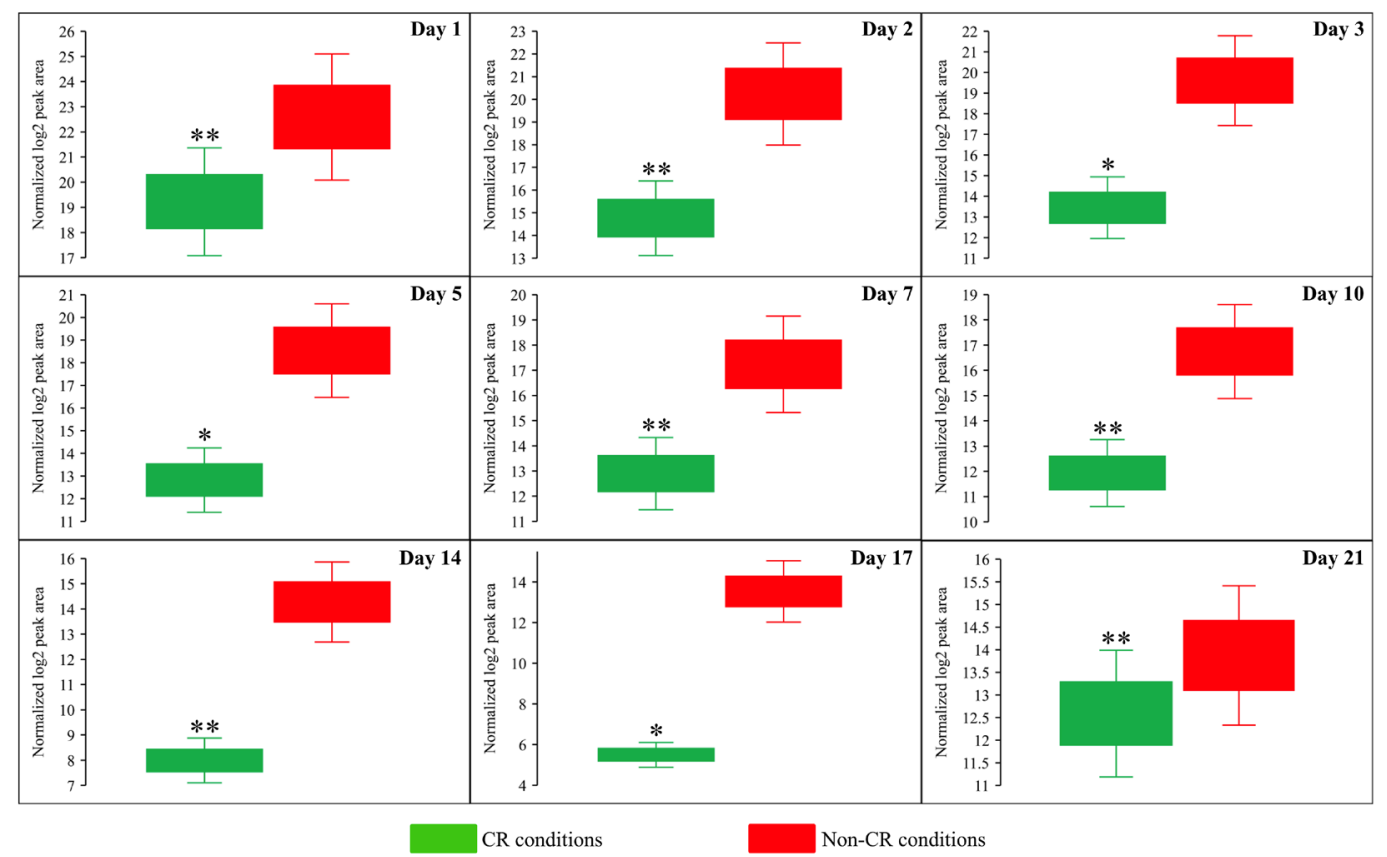

Figure 7: CR significantly decreases the intracellular concentration of $\boldsymbol{S}$-adenosylmethionine (Sam) in yeast cells recovered on any day of culturing. WT strain BY4742 was cultured in the nutrient-rich YP medium initially containing $2 \%$ (w/v) glucose (non-CR conditions) or $0.2 \%(\mathrm{w} / \mathrm{v})$ glucose (CR conditions). Cell aliquots were collected and the metabolomic analysis by LC$\mathrm{MS} / \mathrm{MS}$ was performed as described in the legend for Figure 5. The concentrations of Sam within WT cells cultured under CR or non-CR conditions are shown as the normalized $\log _{2}$ values of mass spectrometric peak areas for Sam. The $p$ values for comparing the means of two groups were calculated using an unpaired two-tailed $t$ test described in Materials and Methods. ${ }^{*} p<0.05,{ }^{* *} p<0.01$ and ${ }^{* * * *} p<0.001$. Data of 2 independent experiments, each being performed twice, are presented.

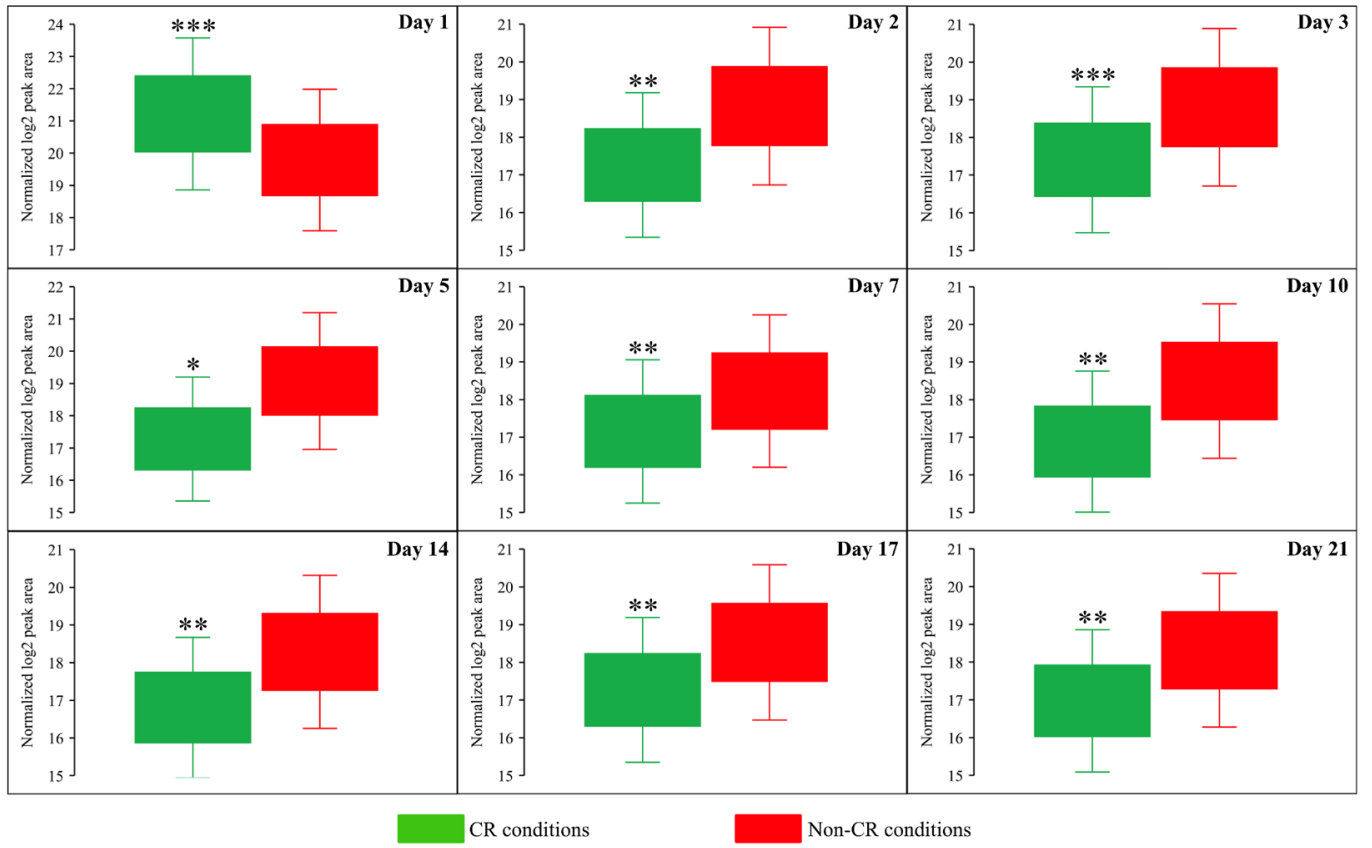

Figure 8: CR significantly decreases the intracellular concentration of methionine (Met) during most days of yeast cell culturing. WT strain BY4742 was cultured in the nutrient-rich YP medium initially containing 2\% (w/v) glucose (non-CR conditions) or $0.2 \%(\mathrm{w} / \mathrm{v})$ glucose (CR conditions). Cell aliquots were collected and the metabolomic analysis by LC-MS/MS was performed as described in the legend for Figure 5. The concentrations of Met within WT cells cultured under CR or non-CR conditions are shown as the normalized $\log _{2}$ values of mass spectrometric peak areas for Met. The $p$ values for comparing the means of two groups were calculated using an unpaired two-tailed $t$ test described in Materials and Methods. ${ }^{*} p<0.05,{ }^{* *} p<0.01$ and ${ }^{* * *} p<0.001$. Data of 2 independent experiments, each being performed twice, are presented. 


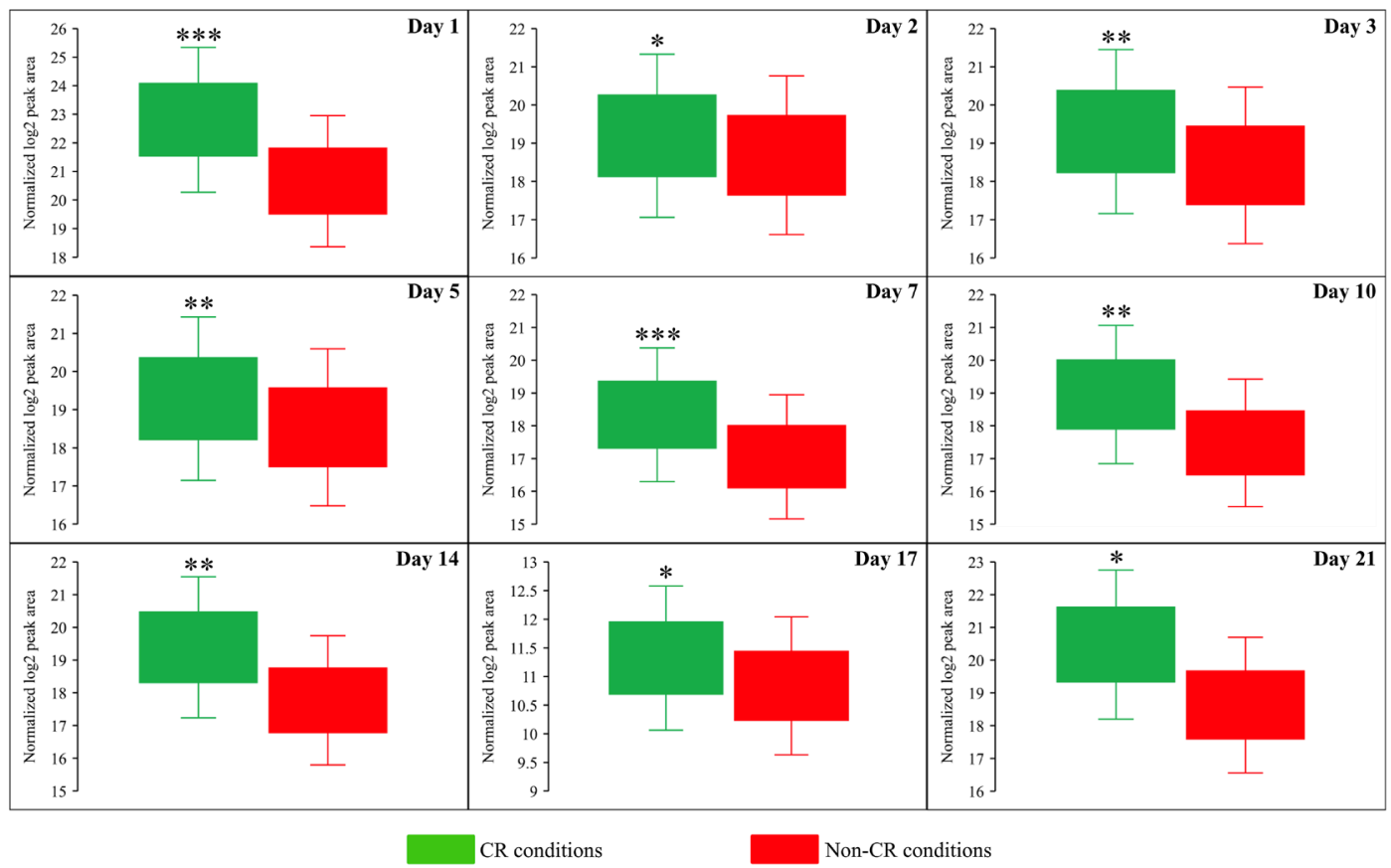

Figure 9: CR significantly increases the intracellular concentration of aspartate (Asp) throughout the entire chronological lifespan of budding yeast. WT strain BY4742 was cultured in the nutrient-rich YP medium initially containing $2 \%$ $(\mathrm{w} / \mathrm{v})$ glucose (non-CR conditions) or $0.2 \%$ (w/v) glucose (CR conditions). Cell aliquots were collected and the metabolomic analysis by LC-MS/MS was performed as described in the legend for Figure 5. The concentrations of Asp within WT cells cultured under CR or nonCR conditions are shown as the normalized $\log _{2}$ values of mass spectrometric peak areas for Asp. The $p$ values for comparing the means of two groups were calculated using an unpaired two-tailed $t$ test described in Materials and Methods. ${ }^{*} p<0.05,{ }^{* *} p<0.01$ and ${ }^{* * *} p<0.001$. Data of 2 independent experiments, each being performed twice, are presented.

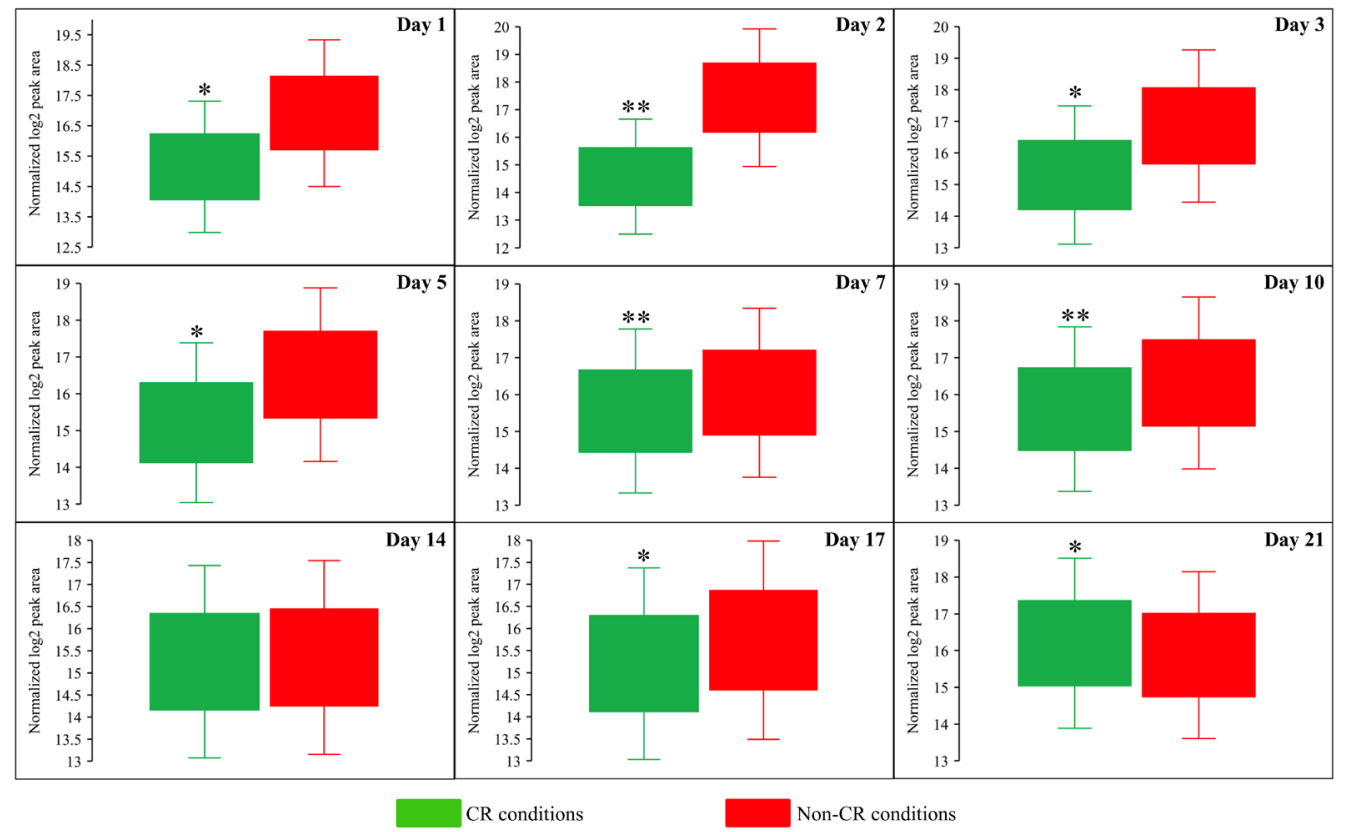

Figure 10: CR significantly decreases the intracellular concentration of cysteine (Cys) during most days of yeast cell culturing. WT strain BY4742 was cultured in the nutrient-rich YP medium initially containing $2 \%(\mathrm{w} / \mathrm{v})$ glucose (non-CR conditions) or $0.2 \%(\mathrm{w} / \mathrm{v})$ glucose (CR conditions). Cell aliquots were collected and the metabolomic analysis by LC-MS/MS was performed as described in the legend for Figure 5. The concentrations of Cys within WT cells cultured under CR or non-CR conditions are shown as the normalized $\log _{2}$ values of mass spectrometric peak areas for Cys. The $p$ values for comparing the means of two groups were calculated using an unpaired two-tailed $t$ test described in Materials and Methods. ${ }^{*} p<0.05,{ }^{* *} p<0.01$. Data of 2 independent experiments, each being performed twice, are presented. 
in intracellular Sam concentration can weaken the proaging Tor1 pathway because it suppresses the protein phosphatase Ppa2p (inorganic pyrophosphatase 2; a known upstream activator of Tor1) [72]. Moreover, a decline in the intracellular concentrations of Met and Cys can slow the pro-aging process of protein synthesis because these two sulfur amino acids promote a thiolation-driven tRNA activation [73].

The observed CR-specific remodeling of adenosine phosphate nucleotide metabolism is also a likely contributor to yeast chronological aging delay by $\mathrm{CR}$. Indeed, the increases in the intracellular ADP:ATP and AMP:ATP ratios are known to correlate with the activating phosphorylation (or inversely correlate with inactivating dephosphorylation) of the anti-aging heterotrimeric protein complex Snf1 (sucrose non-fermenting complex 1) [74]. These in vivo effects of the ADP:ATP and AMP:ATP ratios are not due to AMP or ATP binding to Snf1 [75, 76]. It is feasible that the ADP:ATP and AMP:ATP ratios regulate $\mathrm{Snf1}$ indirectly, either by promoting the activating phosphorylation of Snf1 by the protein kinases Sak1, Tos3 and Elm1 or by suppressing the inactivating dephosphorylation of Snf1 by the protein phosphatase Glc7 [77]. An adenosine phosphate nucleotide can also regulate Snfl directly. Indeed, ADP binding to Snf1 is known to protect it from inactivating dephosphorylation $[75,76]$. As a member of the family of AMP-activated protein kinases [78], Snf1 is a key energy-sensing regulator that phosphorylates and activates or inactivates several protein targets known for their essential roles in defining yeast CLS [77-80].

These findings suggest a hypothetical model of how the observed CR-specific remodeling of cellular metabolism delays the chronological aging of yeast
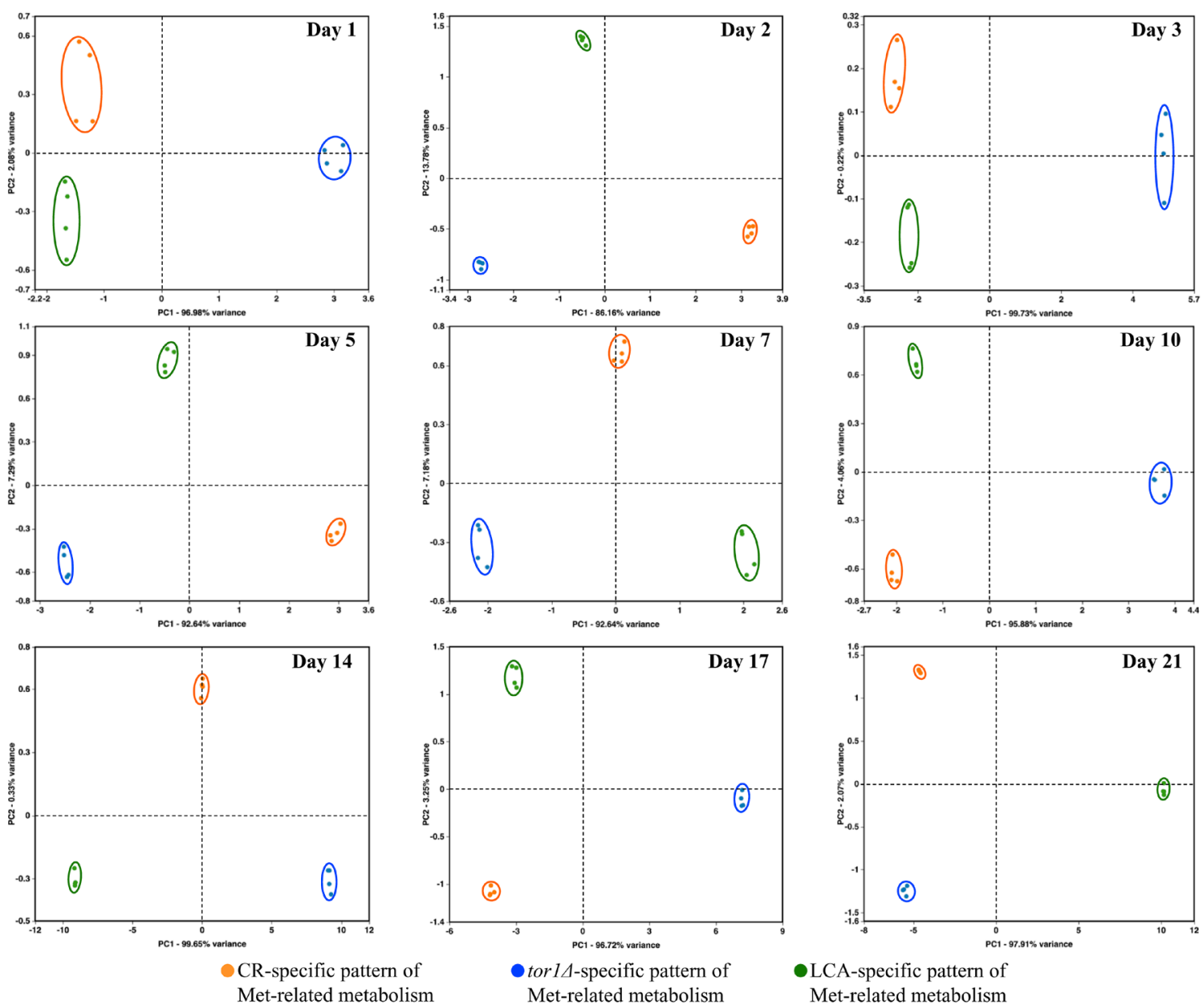

Figure 11: CR, tor1A and LCA differently affect the metabolic pathway for sulfur amino acid biosynthesis on various days of culturing. The WT and tor $1 \Delta$ mutant strains were cultured, cell aliquots were collected and the metabolomic analysis by LC-MS/ MS was performed as described in the legend for Figure 5. A WT strain culture that initially contained 2\% (w/v) glucose served as a control non-CR culture for defining the metabolic patterns created by the CR and tor $1 \Delta$ geroprotectors. A WT strain culture that initially contained $0.2 \%(\mathrm{w} / \mathrm{v})$ glucose without LCA served as a control CR culture for defining the metabolic pattern created by the LCA geroprotector. The data on the relative concentrations of all four metabolites (i.e., Asp, Cys, Met and Sam) within the sulfur amino acid biosynthetic pathway were normalized in age-matched cells. Multivariate analysis by PCA was then used to compare how the CR, tor $1 \Delta$ and LCA geroprotectors affect the metabolic pathway for sulfur amino acid biosynthesis on various days of culturing. Data of 2 independent experiments, each being performed twice, are presented. 


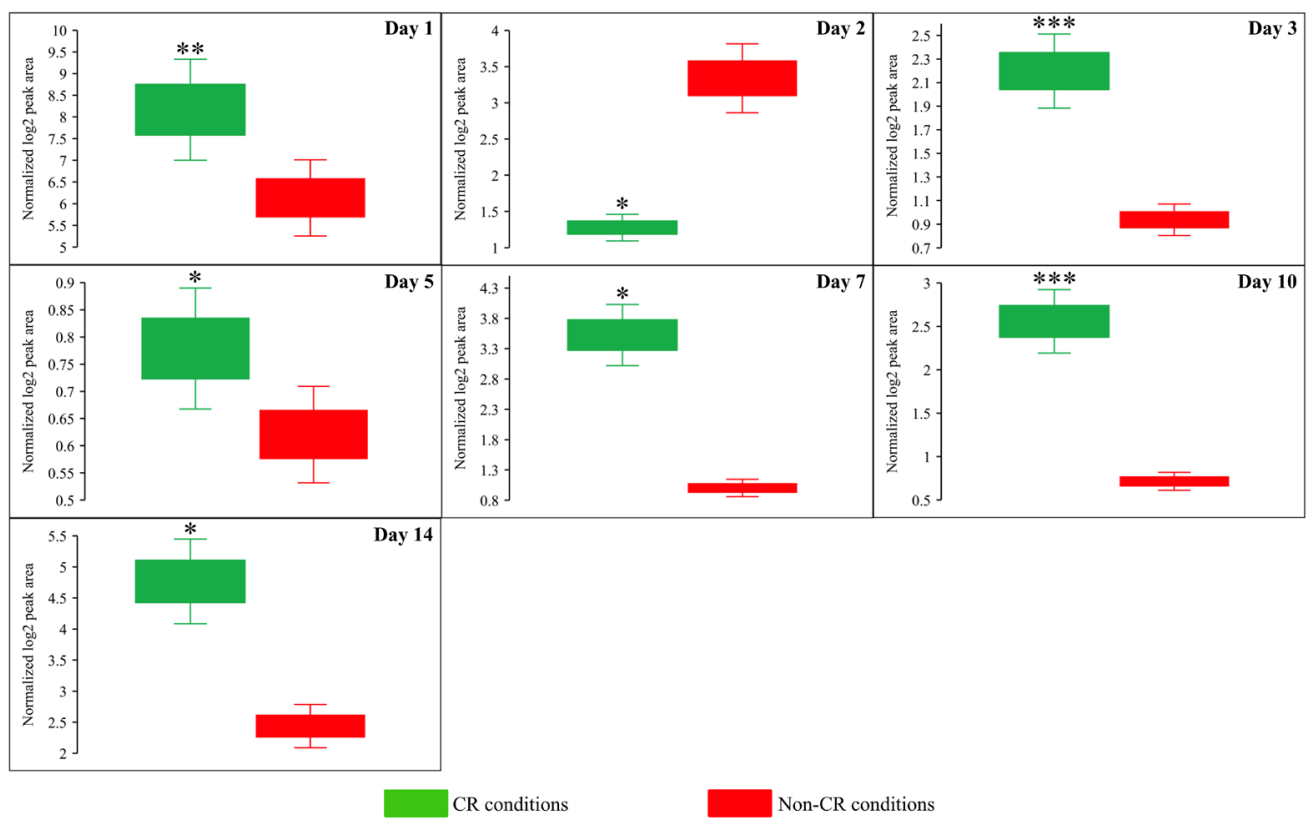

Figure 12: CR significantly increases the AMP:ATP ratios during most days of yeast cell culturing. WT strain BY4742 was cultured in the nutrient-rich YP medium initially containing $2 \%(\mathrm{w} / \mathrm{v}$ ) glucose (non-CR conditions) or $0.2 \%$ (w/v) glucose (CR conditions). Cell aliquots were collected and the metabolomic analysis by LC-MS/MS was performed as described in the legend for Figure 5. The AMP:ATP ratios within WT cells cultured under CR or non-CR conditions are shown as the normalized $\log _{2}$ values of mass spectrometric peak areas for AMP and ATP. The AMP:ATP ratios were not calculated for yeast recovered on days 17 and 21 of culturing because ATP was not detected in WT cells cultured under CR or non-CR conditions without LCA. The $p$ values for comparing the means of two groups were calculated using an unpaired two-tailed $t$ test described in Materials and Methods. ${ }^{*} p<0.05,{ }^{* * *} p<0.01,{ }^{* * *} p<0.001$. Data of 2 independent experiments, each being performed twice, are presented.

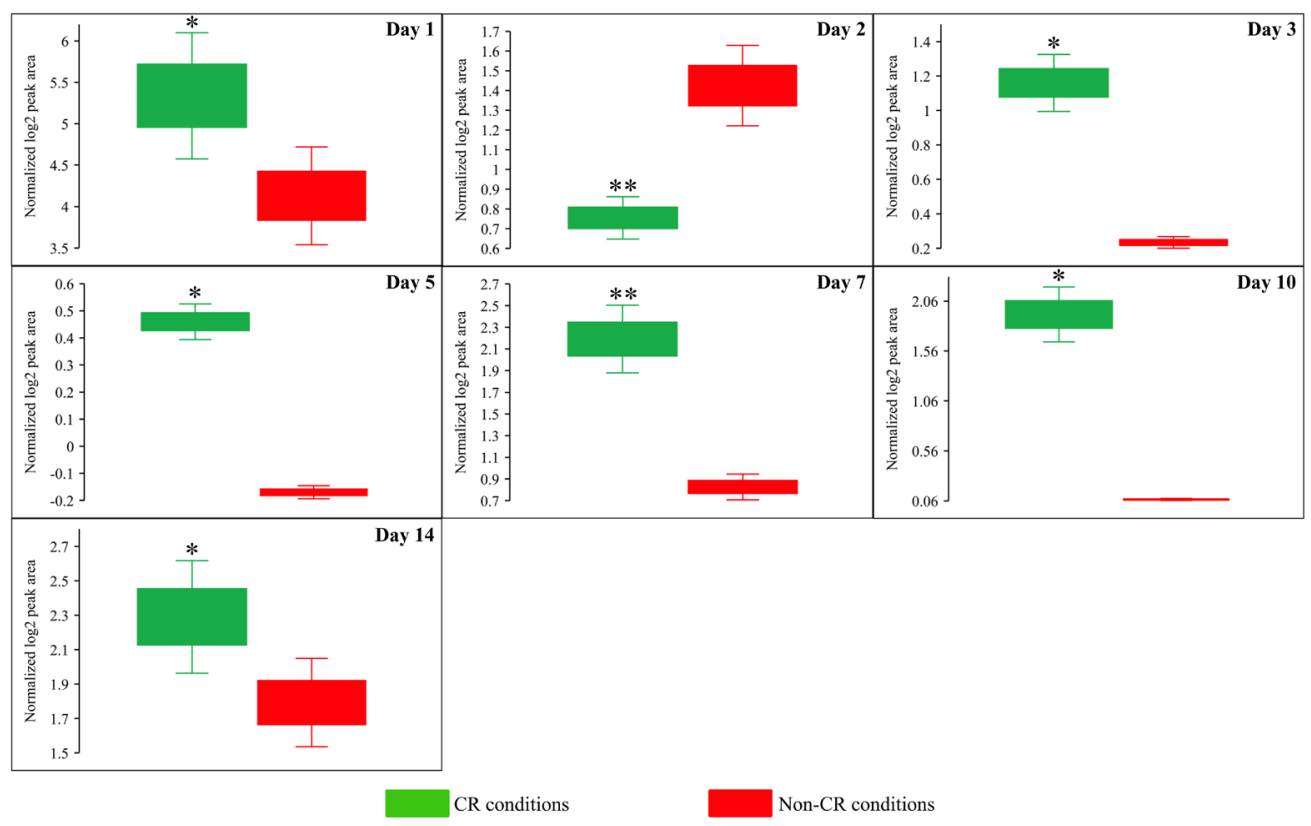

Figure 13: CR significantly increases the ADP:ATP ratios during most days of yeast cell culturing. WT strain BY4742 was cultured in the nutrient-rich YP medium initially containing $2 \%(\mathrm{w} / \mathrm{v}$ ) glucose (non-CR conditions) or $0.2 \%$ (w/v) glucose (CR conditions). Cell aliquots were collected and the metabolomic analysis by LC-MS/MS was performed as described in the legend for Figure 5. The ADP:ATP ratios within WT cells cultured under CR or non-CR conditions are shown as the normalized $\log _{2}$ values of mass spectrometric peak areas for ADP and ATP. The ADP:ATP ratios were not calculated for yeast recovered on days 17 and 21 of culturing because ATP was not detected in WT cells cultured under CR or non-CR conditions without LCA. The $p$ values for comparing the means of two groups were calculated using an unpaired two-tailed $t$ test described in Materials and Methods. ${ }^{*} p<0.05,{ }^{* *} p<0.01$. Data of 2 independent experiments, each being performed twice, are presented. 
cultured in the nutrient-rich YP medium. The model is schematically depicted in Figure 14. The key aspects of this model are as follows: 1) a life-long decline in the intracellular concentrations of Cys and Met weakens tRNA thiolation, thus slowing down the pro-aging process of protein synthesis, 2) a decrease of intracellular Met throughout the chronological lifespan attenuates a direct Met-driven stimulation of the pro-aging Tor1 pathway, thereby lowering the inhibitory effect of Tor1 on autophagy and other anti-aging processes, 3) a deterioration in intracellular Met concentration at diverse stages of chronological aging also weakens a Metdependent suppression of the proteasomal degradation of damaged and dysfunctional proteins, a known anti-aging process, 4) a decline in Sam concentration throughout the chronological lifespan lowers the ability of the protein phosphatase Ppa2p to stimulate the pro-aging Tor1 pathway, and 5) a rise in the ADP:ATP and AMP:ATP ratios on most days of yeast chronological lifespan indirectly (i.e., independent of AMP or ATP binding to Snf1) stimulates the anti-aging protein kinase complex Snf1; Snf1 can also be activated directly, via an ADP binding-dependent protection of Snfl from inactivating dephosphorylation.

This study created two important questions. First, what is the mechanism responsible for ATP decline under CR conditions? We hypothesize that CR might affect the transcription and/or translation of enzymes involved in ATP synthesis and/or degradation in the cytosol, mitochondria or other cellular locations. Of note, a transcription/translation-based mechanism of suppressing methionine biosynthetic enzymes and transporters underlies the ability of CR to lower intracellular methionine and extend yeast RLS [70]. Second, what are the metabolic changes underlying the extremely efficient longevity extension in yeast culture under CR conditions with LCA? Our findings indicate that LCA applied under CR conditions "overrides" the CR-specific metabolic profile of aging delay. We still do not understand the nature of such LCA-specific overriding. Nonetheless, this observation further supports the notion that metabolism is an essential contributor to eukaryotes' aging and longevity.

The challenge for the future is to test the model shown in Figure 14. Our ongoing studies address this challenge. In the future, it would also be interesting to define the metabolic "signatures" specific for other geroprotectors, including the tor $1 \triangle$ mutation and LCA.

\section{MATERIALS AND METHODS}

\section{Yeast strains, media and growth conditions}

The wild-type (WT) strain Saccharomyces

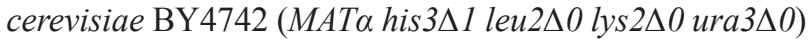
from Thermo Scientific/Open Biosystems was grown in YP medium (1\% yeast extract, $2 \%$ peptone, both from
Fisher Scientific) initially containing the following: 1) $2 \%$ $(\mathrm{w} / \mathrm{v})$ glucose (Fisher Scientific) as a carbon source, 2) $0.2 \%(\mathrm{w} / \mathrm{v})$ glucose as a carbon source or 3$) 0.2 \%(\mathrm{w} / \mathrm{v})$ glucose as a carbon source and $50 \mu \mathrm{M}$ lithocholic acid (LCA). The tor $1 \Delta$ single-gene-deletion mutant strain in the BY4742 genetic background from Thermo Scientific/ Open Biosystems was grown in YP medium initially containing $2 \%(\mathrm{w} / \mathrm{v})$ glucose as a carbon source. Cells were cultured at $30^{\circ} \mathrm{C}$ with rotational shaking at $200 \mathrm{rpm}$ in Erlenmeyer flasks at a "flask volume/medium volume" ratio of $5: 1$.

\section{Cell quenching for metabolite extraction}

Cell aliquots for metabolite extraction were collected on days $1,2,3,5,7,10,14,17$ and 21 of culturing. After measuring the cell titer with the help of a hemocytometer, a volume of the culture that contains the total number of $5.0 \times 10^{8}$ cells was transferred into a precooled at $-5^{\circ} \mathrm{C} 500-\mathrm{ml}$ centrifuge bottle for a Beckman Coulter centrifuge. The centrifuge bottle containing the cells was filled up to the volume of $200 \mathrm{ml}$ with a quenching solution $(60 \%$ methanol in $155 \mathrm{mM}$ ammonium bicarbonate $(\mathrm{ABC})$ buffer, $\mathrm{pH}=8.0$ ) stored at $-20^{\circ} \mathrm{C}$; methanol of a liquid chromatography-mass spectrometry (LC-MS) grade was used. The bottle was centrifuged in a high-speed Beckman Coulter centrifuge at 11,325 $\times \mathrm{g}$ for $3 \mathrm{~min}$ at $-5^{\circ} \mathrm{C}$. The bottle was quickly and tenderly recovered from the centrifuge. The bottle's lid was gently unscrewed, and the supernatant was removed without disturbing the pellet of quenched cells. The cell pellet in each tube was resuspended in $10 \mathrm{ml}$ of ice-cold ABC buffer. The suspension was transferred into a 15-ml highspeed glass centrifuge tube with a polytetrafluoroethylenelined cap (PYREX) for metabolite extraction. Quenched cells were collected by centrifugation in a clinical centrifuge at $3,000 \times \mathrm{g}$ for $3 \mathrm{~min}$ at $0^{\circ} \mathrm{C}$. The supernatant was promptly removed. The tube with the pelleted quenched cells was kept on dry ice or stored at $-80^{\circ} \mathrm{C}$ until metabolite extraction.

\section{Metabolite extraction from quenched cells}

The following was added to the pelleted quenched cells kept on dry ice or stored at $-80^{\circ} \mathrm{C}$ tube: 1) $2 \mathrm{ml}$ of chloroform stored at $\left.-20^{\circ} \mathrm{C}, 2\right) 1 \mathrm{ml}$ of methanol held at $\left.-20^{\circ} \mathrm{C}, 3\right) 1 \mathrm{ml}$ of ice-cold nanopure water and 4) $200 \mu \mathrm{l}$ of 425-600 $\mu \mathrm{m}$ acid-washed glass beads (Sigma-Aldrich); LC-MS grade chloroform, methanol and nanopure water were used. The tube's mouth was covered with aluminum foil. The tube was placed in a foam tube holder kit with a retainer (Thermo Scientific) and vortexed for $30 \mathrm{~min}$ at $4^{\circ} \mathrm{C}$ to facilitate metabolite extraction. The tube was then incubated for $15 \mathrm{~min}$ on ice to promote protein precipitation and the separation of the upper aqueous from the lower organic phase. The tube was centrifuged 
in a clinical centrifuge at $3,000 \times \mathrm{g}$ for $10 \mathrm{~min}$ at $4^{\circ} \mathrm{C}$ to separate the following three phases efficiently: the upper aqueous phase (which contained water-soluble metabolites), middle phase (which had cell debris and proteins) and lower organic phase (which included mostly lipids). A glass micropipette was used to transfer the upper aqueous phase (whose total volume was $\sim 400 \mu \mathrm{l}$ ) to a 1.5-ml Eppendorf tube containing $800 \mu \mathrm{l}$ of acetonitrile (ACN; Thermo Scientific) stored at $-20^{\circ} \mathrm{C}$. The tube was then centrifuged in a tabletop centrifuge at $13,400 \times \mathrm{g}$ for $10 \mathrm{~min}$ at $4^{\circ} \mathrm{C}$. The upper portion of a liquid in the tube (a total volume of this portion was $800 \mu \mathrm{l}$ ) was transferred to an MS vial. This vial was stored at $0^{\circ} \mathrm{C}$ until we used LC-MS/MS to analyze the sample.

\section{LC-MS/MS analysis of extracted metabolites}

LC-MS/MS data were acquired using an Agilent 1100 HPLC system (Agilent Technologies, CA, USA) interfaced with an LTQ Orbitrap Velos mass spectrometer (Thermo Fisher Scientific, Waltham, MA, USA).

Before the sample of extracted metabolites stored in an MS vial at $0^{\circ} \mathrm{C}$ was analyzed by LC, it was initially subjected to ultrasonic sonication for $15 \mathrm{~min}$. The MS vial with the sample was then vortexed three times (each time for $10 \mathrm{sec}$ ) at room temperature. LC separation was carried out using a ZIC-pHILIC column (Merck SeQuant, Umeå, Sweden; $150 \times 2.1 \mathrm{~mm}, 5 \mu \mathrm{m}$ particle size). Solvent A (a 95:5 (v/v) mixture of nanopure water with ACN (respectively) containing $20 \mathrm{mM}$ ammonium acetate) and solvent $\mathrm{B}(\mathrm{ACN})$ were used as the mobile phase for LC; LC-MS grade nanopure water and ACN were used. Supplementary Table 1 provides the LC gradient program used for the chromatographic separation of extracted metabolites. The chromatography column was maintained at $45^{\circ} \mathrm{C}$, and the samples were kept at $0^{\circ} \mathrm{C}$ during metabolite separation.

Mass spectrometric analyses of water-soluble metabolites separated by LC were performed with a Thermo Orbitrap Velos mass spectrometer equipped with a heated electrospray ionization ion source (Thermo Scientific). The mass spectrometer's analyzer was used for primary ions (MS1). Secondary ions (MS2) were identified with the mass spectrometer's detector. Supplementary Tables 2 and 3 show the settings used for the data-dependent acquisition of MS1 and MS2 ions, respectively. We used a sample volume of $10 \mu \mathrm{l}$ for the injection in both the positive and negative ionization modes.

\section{Metabolite identification and quantitation using the LC-MS/MS raw data}

The extracted water-soluble metabolites were identified and quantified with the Compound Discoverer 3.1 software (Fisher Scientific). This software processes raw LC-MS/MS files; it uses MS1 for metabolite quantitation and MS2 for metabolite identification. We used a freely available online library of databases and spectra (https://www.mzcloud.org) to search for MS2 spectra of the raw data.

\section{Miscellaneous procedures}

Statistical analysis was performed using Microsoft Excel's Analysis ToolPack-VBA. All data on cell survival

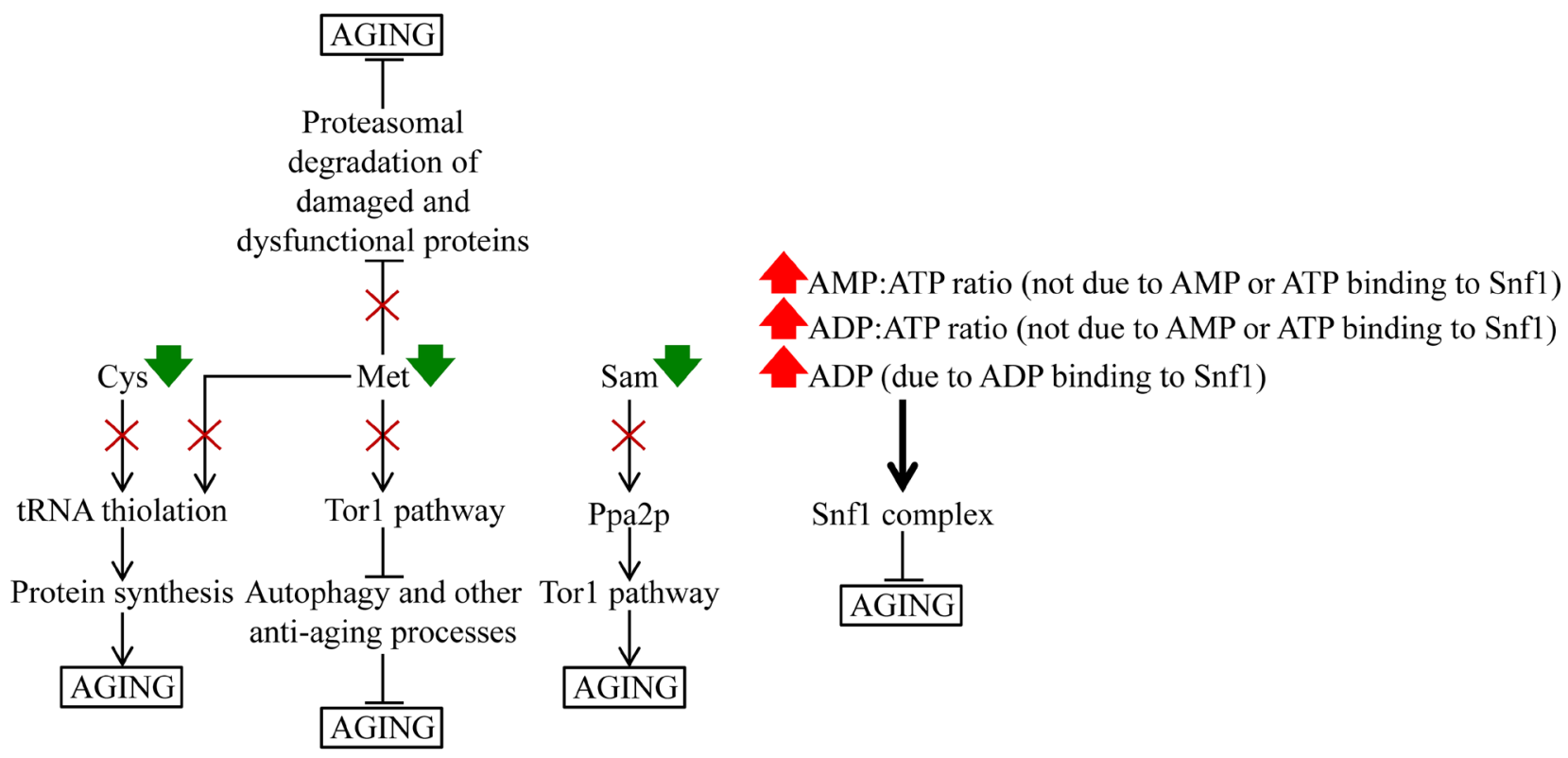

Figure 14: A hypothetical model of how a specific remodeling of cellular metabolism by CR slows down yeast chronological aging. See the text for more details. Abbreviations: Cys, cysteine; Met, methionine; Ppa2p, inorganic pyrophosphatase 2; Sam, $S$-adenosylmethionine; Snf1, sucrose non-fermenting complex 1; Tor1, target of rapamycin complex 1. 
are presented as mean \pm SEM. The $p$ values for comparing the means of two groups using an unpaired two-tailed $t$-test were calculated with the GraphPad Prism 7 statistics software. The logrank test for comparing each pair of survival curves was performed with GraphPad Prism 7. Two survival curves were considered statistically different if the $p$ value was less than 0.05 . The BioVinci software (https://vinci.bioturing.com/) was used to generate the 2-D plots by PCA of the metabolomic data.

\section{Abbreviations}

ABC: ammonium bicarbonate; $\mathrm{ACN}$ : acetonitrile; CLS: chronological lifespan; CR: caloric restriction; LCA: lithocholic acid; LC: liquid chromatography; MS: mass spectrometry; MS1: primary ion in tandem mass spectrometry; MS2: secondary ion in tandem mass spectrometry; PCA: principal component analysis; Sam: $S$-adenosylmethionine; WT: wild type; 5-Mtf: 5-methyltetrahydrofolate.

\section{Author contributions}

VT and KM contributed to the study concept and design. KM and VT contributed to the acquisition, analysis, and interpretation of data. KM and VT drafted the manuscript. All authors read, revised and approved the final manuscript.

\section{ACKNOWLEDGMENTS}

We acknowledge the Centre for Biological Applications of Mass Spectrometry at Concordia University for outstanding services.

\section{CONFLICTS OF INTEREST}

Authors have no conflicts of interest to declare.

\section{FUNDING}

This study was supported by grants from the Natural Sciences and Engineering Research Council of Canada (RGPIN 2014-04482 and CRDPJ 515900-17). K.M. was supported by the Concordia University Armand C. Archambault Fellowship and the Concordia University Dean of Arts and Sciences Award of Excellence.

\section{REFERENCES}

1. Eisenberg T, Knauer H, Schauer A, Büttner S, Ruckenstuhl C, Carmona-Gutierrez D, Ring J, Schroeder S, Magnes C, Antonacci L, Fussi H, Deszcz L, Hartl R, et al. Induction of autophagy by spermidine promotes longevity. Nat Cell Biol. 2009; 11:1305-14. https://doi.org/10.1038/ncb1975. [PubMed]
2. Fuchs S, Bundy JG, Davies SK, Viney JM, Swire JS, Leroi AM. A metabolic signature of long life in Caenorhabditis elegans. BMC Biol. 2010; 8:14. https://doi. org/10.1186/1741-7007-8-14. [PubMed]

3. Yoshida R, Tamura T, Takaoka C, Harada K, Kobayashi A, Mukai Y, Fukusaki E. Metabolomics-based systematic prediction of yeast lifespan and its application for semirational screening of ageing-related mutants. Aging Cell. 2010; 9:616-25. https://doi.org/10.1111/j.14749726.2010.00590.x. [PubMed]

4. Houtkooper RH, Argmann C, Houten SM, Cantó C, Jeninga EH, Andreux PA, Thomas C, Doenlen R, Schoonjans K, Auwerx J. The metabolic footprint of aging in mice. Sci Rep. 2011; 1:134. https://doi.org/10.1038/srep00134. [PubMed]

5. Sarup P, Pedersen SM, Nielsen NC, Malmendal A, Loeschcke V. The metabolic profile of long-lived Drosophila melanogaster. PLoS One. 2012; 7:e47461. https://doi.org/10.1371/journal.pone.0047461. [PubMed]

6. Butler JA, Mishur RJ, Bhaskaran S, Rea SL. A metabolic signature for long life in the Caenorhabditis elegans Mit mutants. Aging Cell. 2013; 12:130-38. https://doi. org/10.1111/acel.12029. [PubMed]

7. Gonzalez-Covarrubias V, Beekman M, Uh HW, Dane A, Troost J, Paliukhovich I, van der Kloet FM, HouwingDuistermaat J, Vreeken RJ, Hankemeier T, Slagboom EP. Lipidomics of familial longevity. Aging Cell. 2013; 12:42634. https://doi.org/10.1111/acel.12064. [PubMed]

8. Tomás-Loba A, Bernardes de Jesus B, Mato JM, Blasco MA. A metabolic signature predicts biological age in mice. Aging Cell. 2013; 12:93-101. https://doi.org/10.1111/ acel.12025. [ubMed]

9. Avanesov AS, Ma S, Pierce KA, Yim SH, Lee BC, Clish $\mathrm{CB}$, Gladyshev VN. Age- and diet-associated metabolome remodeling characterizes the aging process driven by damage accumulation. Elife. 2014; 3:e02077. https://doi. org/10.7554/eLife.02077. [PubMed]

10. Hertel J, Friedrich N, Wittfeld K, Pietzner M, Budde K, Van der Auwera S, Lohmann T, Teumer A, Völzke H, Nauck M, Grabe HJ. Measuring Biological Age via Metabonomics: The Metabolic Age Score. J Proteome Res. 2016; 15: 400-10. https://doi.org/10.1021/acs.jproteome.5b00561. [PubMed]

11. Wiley CD, Campisi J. From Ancient Pathways to Aging Cells-Connecting Metabolism and Cellular Senescence. Cell Metab. 2016; 23:1013-21. https://doi.org/10.1016/j. cmet.2016.05.010. [PubMed]

12. Gao AW, Chatzispyrou IA, Kamble R, Liu YJ, Herzog K, Smith RL, van Lenthe H, Vervaart MA, van Cruchten A, Luyf AC, van Kampen A, Pras-Raves ML, Vaz FM, Houtkooper RH. A sensitive mass spectrometry platform identifies metabolic changes of life history traits in C. elegans. Sci Rep. 2017; 7:2408. https://doi.org/10.1038/ s41598-017-02539-w. [PubMed]

13. Johnson LC, Martens CR, Santos-Parker JR, Bassett CJ, Strahler TR, Cruickshank-Quinn C, Reisdorph N, McQueen 
MB, Seals DR. Amino acid and lipid associated plasma metabolomic patterns are related to healthspan indicators with ageing. Clin Sci (Lond). 2018; 132:1765-77. https:// doi.org/10.1042/CS20180409. [PubMed]

14. Ma Z, Wang H, Cai Y, Wang H, Niu K, Wu X, Ma H, Yang Y, Tong W, Liu F, Liu Z, Zhang Y, Liu R, et al. Epigenetic drift of H3K27me3 in aging links glycolysis to healthy longevity in Drosophila. Elife. 2018; 7:e35368. https://doi. org/10.7554/eLife.35368. [PubMed]

15. Bunning BJ, Contrepois K, Lee-McMullen B, Dhondalay GK, Zhang W, Tupa D, Raeber O, Desai M, Nadeau KC, Snyder MP, Andorf S. Global metabolic profiling to model biological processes of aging in twins. Aging Cell. 2020; 19:e13073. https://doi.org/10.1111/acel.13073. [PubMed]

16. D’Antona G, Ragni M, Cardile A, Tedesco L, Dossena M, Bruttini F, Caliaro F, Corsetti G, Bottinelli R, Carruba MO, Valerio A, Nisoli E. Branched-chain amino acid supplementation promotes survival and supports cardiac and skeletal muscle mitochondrial biogenesis in middleaged mice. Cell Metab. 2010; 12:362-72. https://doi. org/10.1016/j.cmet.2010.08.016. [PubMed]

17. Peng W, Robertson L, Gallinetti J, Mejia P, Vose S, Charlip A, Chu T, Mitchell JR. Surgical stress resistance induced by single amino acid deprivation requires Gcn2 in mice. Sci Transl Med. 2012; 4:118ra11. https://doi.org/10.1126/ scitranslmed.3002629. [PubMed]

18. Sanchez-Roman I, Barja G. Regulation of longevity and oxidative stress by nutritional interventions: role of methionine restriction. Exp Gerontol. 2013; 48:1030-42. https://doi.org/10.1016/j.exger.2013.02.021. [PubMed]

19. Galluzzi L, Pietrocola F, Levine B, Kroemer G. Metabolic control of autophagy. Cell. 2014; 159:1263-76. https://doi. org/10.1016/j.cell.2014.11.006. [PubMed]

20. Jové M, Naudí A, Ramírez-Núñez O, Portero-Otín M, Selman C, Withers DJ, Pamplona R. Caloric restriction reveals a metabolomic and lipidomic signature in liver of male mice. Aging Cell. 2014; 13:828-37. https://doi. org/10.1111/acel.12241. [PubMed]

21. Solon-Biet SM, McMahon AC, Ballard JW, Ruohonen $\mathrm{K}$, Wu LE, Cogger VC, Warren A, Huang X, Pichaud N, Melvin RG, Gokarn R, Khalil M, Turner N, et al. The ratio of macronutrients, not caloric intake, dictates cardiometabolic health, aging, and longevity in ad libitumfed mice. Cell Metab. 2014; 19:418-30. https://doi. org/10.1016/j.cmet.2014.02.009. [PubMed]

22. Finkel T. The metabolic regulation of aging. Nat Med. 2015; 21:1416-23. https://doi.org/10.1038/nm.3998. [PubMed]

23. Fontana L, Partridge L. Promoting health and longevity through diet: from model organisms to humans. Cell. 2015; 161:106-18. https://doi.org/10.1016/j.cell.2015.02.020. [PubMed]

24. Hine C, Harputlugil E, Zhang Y, Ruckenstuhl C, Lee BC, Brace L, Longchamp A, Treviño-Villarreal JH, Mejia P, Ozaki CK, Wang R, Gladyshev VN, Madeo F, et al. Endogenous hydrogen sulfide production is essential for dietary restriction benefits. Cell. 2015; 160:132-44. https:// doi.org/10.1016/j.cell.2014.11.048. [PubMed]

25. Madeo F, Zimmermann A, Maiuri MC, Kroemer G. Essential role for autophagy in life span extension. J Clin Invest. 2015; 125:85-93. https://doi.org/10.1172/JCI73946. [PubMed]

26. Bárcena C, Quirós PM, Durand S, Mayoral P, Rodríguez F, Caravia XM, Mariño G, Garabaya C, Fernández-García MT, Kroemer G, Freije JM, López-Otín C. Methionine Restriction Extends Lifespan in Progeroid Mice and Alters Lipid and Bile Acid Metabolism. Cell Rep. 2018; 24:2392-403. https://doi. org/10.1016/j.celrep.2018.07.089. [PubMed]

27. Brandhorst S, Longo VD. Protein Quantity and Source, Fasting-Mimicking Diets, and Longevity. Adv Nutr. 2019; 10 (suppl 4):S340-S350. https://doi.org/10.1093/advances/ nmz079. [PubMed]

28. Canfielda CA, Bradshaw PC. Amino acids in the regulation of aging and aging-related diseases. Transl Med Aging. 2019; 3:70-89. https://doi.org/10.1016/j.tma.2019.09.001.

29. Green CL, Lamming DW. Regulation of metabolic health by essential dietary amino acids. Mech Ageing Dev. 2019; 177:186-200. https://doi.org/10.1016/j.mad.2018.07.004. [PubMed]

30. Kitada M, Ogura Y, Monno I, Koya D. The impact of dietary protein intake on longevity and metabolic health. EBioMedicine. 2019; 43:632-40. https://doi.org/10.1016/j. ebiom.2019.04.005. [PubMed]

31. Liu YJ, Janssens GE, McIntyre RL, Molenaars M, Kamble R, Gao AW, Jongejan A, Weeghel MV, MacInnes AW, Houtkooper RH. Glycine promotes longevity in Caenorhabditis elegans in a methionine cycle-dependent fashion. PLoS Genet. 2019; 15:e1007633. https://doi. org/10.1371/journal.pgen.1007633. [PubMed]

32. Mitchell SJ, Bernier M, Mattison JA, Aon MA, Kaiser TA, Anson RM, Ikeno Y, Anderson RM, Ingram DK, de Cabo R. Daily Fasting Improves Health and Survival in Male Mice Independent of Diet Composition and Calories. Cell Metab. 2019; 29:221-228.e3. https://doi.org/10.1016/j. cmet.2018.08.011. [PubMed]

33. Aon MA, Bernier M, Mitchell SJ, Di Germanio C, Mattison JA, Ehrlich MR, Colman RJ, Anderson RM, de Cabo R. Untangling Determinants of Enhanced Health and Lifespan through a Multi-omics Approach in Mice. Cell Metab. 2020; 32:100-116.e4. https://doi.org/10.1016/j.cmet.2020.04.018. [PubMed]

34. Giese GE, Walker MD, Ponomarova O, Zhang H, Li X, Minevich G, Walhout AJ. Caenorhabditis elegans methionine/S-adenosylmethionine cycle activity is sensed and adjusted by a nuclear hormone receptor. Elife. 2020; 9:e60259. https://doi.org/10.7554/eLife.60259. [PubMed]

35. Hansen M, Hsu AL, Dillin A, Kenyon C. New genes tied to endocrine, metabolic, and dietary regulation of lifespan from a Caenorhabditis elegans genomic RNAi screen. PLoS Genet. 2005; 1:119-28. https://doi.org/10.1371/journal. pgen.0010017. [PubMed] 
36. Bitto A, Wang AM, Bennett CF, Kaeberlein M. Biochemical Genetic Pathways that Modulate Aging in Multiple Species. Cold Spring Harb Perspect Med. 2015; 5:a025114. https:// doi.org/10.1101/cshperspect.a025114. [PubMed]

37. Passarino G, De Rango F, Montesanto A. Human longevity: genetics or Lifestyle? It takes two to tango. Immun Ageing. 2016; 13:12. https://doi.org/10.1186/s12979-016-0066-Z. [PubMed]

38. Dato S, Rose G, Crocco P, Monti D, Garagnani P, Franceschi C, Passarino G. The genetics of human longevity: an intricacy of genes, environment, culture and microbiome. Mech Ageing Dev. 2017; 165:147-55. https:// doi.org/10.1016/j.mad.2017.03.011. [PubMed]

39. Giuliani C, Garagnani P, Franceschi C. Genetics of Human Longevity Within an Eco-Evolutionary Nature-Nurture Framework. Circ Res. 2018; 123:745-72. https://doi. org/10.1161/CIRCRESAHA.118.312562. [PubMed]

40. Van Raamsdonk JM. Mechanisms underlying longevity: A genetic switch model of aging. Exp Gerontol. 2018; 107:136-39. https://doi.org/10.1016/j.exger.2017.08.005. [PubMed]

41. Costa D, Scognamiglio M, Fiorito C, Benincasa G, Napoli C. Genetic background, epigenetic factors and dietary interventions which influence human longevity. Biogerontology. 2019; 20:605-26. https://doi.org/10.1007/ s10522-019-09824-3. [PubMed]

42. Dall KB, Færgeman NJ. Metabolic regulation of lifespan from a C. elegans perspective. Genes Nutr. 2019; 14:25. https://doi.org/10.1186/s12263-019-0650-x. [PubMed]

43. Morris BJ, Willcox BJ, Donlon TA. Genetic and epigenetic regulation of human aging and longevity. Biochim Biophys Acta Mol Basis Dis. 2019; 1865:1718-44. https://doi. org/10.1016/j.bbadis.2018.08.039. [PubMed]

44. Baur JA, Pearson KJ, Price NL, Jamieson HA, Lerin C, Kalra A, Prabhu VV, Allard JS, Lopez-Lluch G, Lewis K, Pistell PJ, Poosala S, Becker KG, et al. Resveratrol improves health and survival of mice on a high-calorie diet. Nature. 2006; 444:337-42. https://doi.org/10.1038/ nature05354. [PubMed]

45. Liu B, Ghosh S, Yang X, Zheng H, Liu X, Wang Z, Jin G, Zheng B, Kennedy BK, Suh Y, Kaeberlein M, Tryggvason K, Zhou Z. Resveratrol rescues SIRT1-dependent adult stem cell decline and alleviates progeroid features in laminopathy-based progeria. Cell Metab. 2012; 16:738-50. https://doi.org/10.1016/j.cmet.2012.11.007. [PubMed]

46. Gomes AP, Price NL, Ling AJ, Moslehi JJ, Montgomery MK, Rajman L, White JP, Teodoro JS, Wrann CD, Hubbard BP, Mercken EM, Palmeira CM, de Cabo R, et al. Declining $\mathrm{NAD}(+)$ induces a pseudohypoxic state disrupting nuclearmitochondrial communication during aging. Cell. 2013; 155:1624-38. https://doi.org/10.1016/j.cell.2013.11.037. [PubMed]

47. Harrison DE, Strong R, Allison DB, Ames BN, Astle CM, Atamna H, Fernandez E, Flurkey K, Javors MA, Nadon NL, Nelson JF, Pletcher S, Simpkins JW, et al. Acarbose,
17- $\alpha$-estradiol, and nordihydroguaiaretic acid extend mouse lifespan preferentially in males. Aging Cell. 2014; 13:27382. https://doi.org/10.1111/acel.12170. [PubMed]

48. Hernández-Damián J, Andérica-Romero AC, PedrazaChaverri J. Paradoxical cellular effects and biological role of the multifaceted compound nordihydroguaiaretic acid. Arch Pharm (Weinheim). 2014; 347:685-97. https://doi. org/10.1002/ardp.201400159. [PubMed]

49. Weimer S, Priebs J, Kuhlow D, Groth M, Priebe S, Mansfeld J, Merry TL, Dubuis S, Laube B, Pfeiffer AF, Schulz TJ, Guthke R, Platzer M, et al. D-Glucosamine supplementation extends life span of nematodes and of ageing mice. Nat Commun. 2014; 5:3563. https://doi. org/10.1038/ncomms4563. [PubMed]

50. Song YM, Lee YH, Kim JW, Ham DS, Kang ES, Cha BS, Lee HC, Lee BW. Metformin alleviates hepatosteatosis by restoring SIRT1-mediated autophagy induction via an AMP-activated protein kinase-independent pathway. Autophagy. 2015; 11:46-59. https://doi.org/10.4161/15548 627.2014.984271. [PubMed]

51. Walsh ME, Bhattacharya A, Sataranatarajan K, Qaisar R, Sloane L, Rahman MM, Kinter M, Van Remmen H. The histone deacetylase inhibitor butyrate improves metabolism and reduces muscle atrophy during aging. Aging Cell. 2015; 14:957-70. https://doi.org/10.1111/acel.12387. [PubMed]

52. Jamwal S, Kumar P. Spermidine ameliorates 3-nitropropionic acid (3-NP)-induced striatal toxicity: possible role of oxidative stress, neuroinflammation, and neurotransmitters. Physiol Behav. 2016; 155:180-87. https://doi.org/10.1016/j.physbeh.2015.12.015. [PubMed]

53. Lin AL, Jahrling JB, Zhang W, DeRosa N, Bakshi V, Romero P, Galvan V, Richardson A. Rapamycin rescues vascular, metabolic and learning deficits in apolipoprotein E4 transgenic mice with pre-symptomatic Alzheimer's disease. J Cereb Blood Flow Metab. 2017; 37:217-26. https://doi.org/10.1177/0271678X15621575. [PubMed]

54. Katsyuba E, Mottis A, Zietak M, De Franco F, van der Velpen V, Gariani K, Ryu D, Cialabrini L, Matilainen O, Liscio P, Giacchè N, Stokar-Regenscheit N, Legouis D, et al. De novo NAD+ synthesis enhances mitochondrial function and improves health. Nature. 2018; 563:354-59. https://doi.org/10.1038/s41586-018-0645-6. [PubMed]

55. Kezic A, Popovic L, Lalic K. mTOR Inhibitor Therapy and Metabolic Consequences: Where Do We Stand? Oxid Med Cell Longev. 2018; 2018:2640342. https://doi. org/10.1155/2018/2640342. [ [PubMed]

56. Shintani H, Shintani T, Ashida H, Sato M. Calorie Restriction Mimetics: Upstream-Type Compounds for Modulating Glucose Metabolism. Nutrients. 2018; 10:1821. https://doi.org/10.3390/nu10121821. [PubMed]

57. Bridgeman SC, Northrop W, Melton PE, Ellison GC, Newsholme P, Mamotte CDS. Butyrate generated by gut microbiota and its therapeutic role in metabolic syndrome. Pharmacol Res. 2020; 160:105174. https://doi. org/10.1016/i.phrs.2020.105174. [PubMed] 
58. Smith DL Jr, Orlandella RM, Allison DB, Norian LA. Diabetes medications as potential calorie restriction mimetics-a focus on the alpha-glucosidase inhibitor acarbose. Geroscience. 2020. https://doi.org/10.1007/ s11357-020-00278-x. [Epub ahead of print]. [PubMed]

59. López-Otín C, Galluzzi L, Freije JM, Madeo F, Kroemer G. Metabolic control of longevity. Cell. 2016; 166:802-21. https://doi.org/10.1016/j.cell.2016.07.031. [PubMed]

60. Catic A. Cellular Metabolism and Aging. Prog Mol Biol Transl Sci. 2018; 155:85-107. https://doi.org/10.1016/ bs.pmbts.2017.12.003. [PubMed]

61. Goldberg AA, Bourque SD, Kyryakov P, Gregg C, BoukhViner T, Beach A, Burstein MT, Machkalyan G, Richard V, Rampersad S, Cyr D, Milijevic S, Titorenko VI. Effect of calorie restriction on the metabolic history of chronologically aging yeast. Exp Gerontol. 2009; 44:55571. https://doi.org/10.1016/j.exger.2009.06.001. [PubMed]

62. Goldberg AA, Richard VR, Kyryakov P, Bourque SD, Beach A, Burstein MT, Glebov A, Koupaki O, Boukh-Viner T, Gregg C, Juneau M, English AM, Thomas DY, Titorenko VI. Chemical genetic screen identifies lithocholic acid as an anti-aging compound that extends yeast chronological life span in a TOR-independent manner, by modulating housekeeping longevity assurance processes. Aging (Albany NY). 2010; 2:393-414. https://doi.org/10.18632/ aging.100168. [PubMed]

63. Mohammad K, Jiang H, Titorenko VI. Quantitative metabolomics of Saccharomyces cerevisiae using liquid chromatography coupled with tandem mass spectrometry. J Vis Exp. 2021; 166:62061. https://doi.org/10.3791/62061. [PubMed]

64. Thomas D, Surdin-Kerjan Y. Metabolism of sulfur amino acids in Saccharomyces cerevisiae. Microbiol Mol Biol Rev. 1997; 61:503-32. [PubMed]

65. Lee BC, Kaya A, Gladyshev VN. Methionine restriction and life-span control. Ann N Y Acad Sci. 2016; 1363:116-24. https://doi.org/10.1111/nyas.12973. [PubMed]

66. McIsaac RS, Lewis KN, Gibney PA, Buffenstein R. From yeast to human: exploring the comparative biology of methionine restriction in extending eukaryotic life span. Ann N Y Acad Sci. 2016; 1363:155-70. https://doi. org/10.1111/nyas.13032. [PubMed]

67. Ables GP, Johnson JE. Pleiotropic responses to methionine restriction. Exp Gerontol. 2017; 94:83-88. https://doi. org/10.1016/j.exger.2017.01.012. [PubMed]

68. Latimer MN, Freij KW, Cleveland BM, Biga PR. Physiological and molecular mechanisms of methionine restriction. Front Endocrinol (Lausanne). 2018; 9:217. https://doi.org/10.3389/fendo.2018.00217. [PubMed]

69. Parkhitko AA, Jouandin P, Mohr SE, Perrimon N. Methionine metabolism and methyltransferases in the regulation of aging and lifespan extension across species. Aging Cell. 2019; 18:e13034. https://doi.org/10.1111/acel.13034. [PubMed]

70. Zou K, Rouskin S, Dervishi K, McCormick MA, Sasikumar A, Deng C, Chen Z, Kaeberlein M, Brem RB, Polymenis
M, Kennedy BK, Weissman JS, Zheng J, et al. Life span extension by glucose restriction is abrogated by methionine supplementation: cross-talk between glucose and methionine and implication of methionine as a key regulator of life span. Sci Adv. 2020; 6:eaba1306. https:// doi.org/10.1126/sciadv.aba1306. [PubMed]

71. Ruckenstuhl C, Netzberger C, Entfellner I, CarmonaGutierrez D, Kickenweiz T, Stekovic S, Gleixner C, Schmid C, Klug L, Sorgo AG, Eisenberg T, Büttner S, Mariño G, et al. Lifespan extension by methionine restriction requires autophagy-dependent vacuolar acidification. PLoS Genet. 2014; 10:e1004347. https://doi.org/10.1371/journal. pgen.1004347. [PubMed]

72. Sutter BM, Wu X, Laxman S, Tu BP. Methionine inhibits autophagy and promotes growth by inducing the SAMresponsive methylation of PP2A. Cell. 2013; 154:403-15. https://doi.org/10.1016/j.cell.2013.06.041. [PubMed]

73. Laxman S, Sutter BM, Wu X, Kumar S, Guo X, Trudgian DC, Mirzaei H, Tu BP. Sulfur amino acids regulate translational capacity and metabolic homeostasis through modulation of tRNA thiolation. Cell. 2013; 154:416-29. https://doi.org/10.1016/j.cell.2013.06.043. [PubMed]

74. Wilson WA, Hawley SA, Hardie DG. Glucose repression/ derepression in budding yeast: SNF1 protein kinase is activated by phosphorylation under derepressing conditions, and this correlates with a high AMP:ATP ratio. Curr Biol. 1996; 6:1426-34. https://doi.org/10.1016/s09609822(96)00747-6. [PubMed]

75. Chandrashekarappa DG, McCartney RR, Schmidt MC. Subunit and domain requirements for adenylatemediated protection of Snfl kinase activation loop from dephosphorylation. J Biol Chem. 2011; 286:44532-41. https://doi.org/10.1074/jbc.M111.315895. [PubMed]

76. Mayer FV, Heath R, Underwood E, Sanders MJ, Carmena D, McCartney RR, Leiper FC, Xiao B, Jing C, Walker PA, Haire LF, Ogrodowicz R, Martin SR, et al. ADP regulates SNF1, the Saccharomyces cerevisiae homolog of AMPactivated protein kinase. Cell Metab. 2011; 14:707-14. https://doi.org/10.1016/j.cmet.2011.09.009. [PubMed]

77. Conrad M, Schothorst J, Kankipati HN, Van Zeebroeck G, Rubio-Texeira M, Thevelein JM. Nutrient sensing and signaling in the yeast Saccharomyces cerevisiae. FEMS Microbiol Rev. 2014; 38:254-99. https://doi. org/10.1111/1574-6976.12065. [PubMed]

78. Hedbacker $\mathrm{K}$, Carlson M. SNF1/AMPK pathways in yeast. Front Biosci. 2008; 13:2408-20. https://doi. org/10.2741/2854. [PubMed]

79. Shashkova S, Welkenhuysen N, Hohmann S. Molecular communication: crosstalk between the Snfl and other signaling pathways. FEMS Yeast Res. 2015; 15:fov026. https://doi.org/10.1093/femsyr/fov026. [PubMed]

80. Coccetti P, Nicastro R, Tripodi F. Conventional and emerging roles of the energy sensor Snfl/AMPK in Saccharomyces cerevisiae. Microb Cell. 2018; 5:482-94. https://doi.org/10.15698/mic2018.11.655. [PubMed] 